Research Article

\title{
A Partition Spatial Filtering Method for Acoustic Array Configuration
}

\author{
Zhihong Liu, ${ }^{1,2}$ Xilong Zhang $\mathbb{D}^{1,2}$ Zunmin Liu, ${ }^{1,2}$ Chuijie Yi, ${ }^{2}$ and Ming Ma ${ }^{1}$ \\ ${ }^{1}$ School of Mechanical and Automotive Engineering, Qingdao University of Technology, Qingdao 266520, China \\ ${ }^{2}$ Key Laboratory of Ministry of Education of China, Qingdao University of Technology, Qingdao 266520, China \\ Correspondence should be addressed to Xilong Zhang; zhangxilong@qut.edu.cn
}

Received 9 December 2020; Accepted 1 February 2021; Published 11 February 2021

Academic Editor: Rafał Burdzik

Copyright (c) 2021 Zhihong Liu et al. This is an open access article distributed under the Creative Commons Attribution License, which permits unrestricted use, distribution, and reproduction in any medium, provided the original work is properly cited.

Acoustic array is a ubiquitous tool for locating and quantifying sound sources. However, its effectiveness depends greatly on the array configuration. This paper presents an array configuration method to enhance array performance, especially on the spatial resolution and the Doppler effect correction. The problem of array configuration is formulated into a position matrix determined by introducing partition spatial filtering. Irregular coaxial ring grid spacings and partition filtering conditions are suggested to control array spatial resolution. Geometrical parameters and performance indicators are constructed to quantify the relationships between the array configuration and performance. Based on these quantitative relations, the spatial variation of the array beam pattern and the Doppler effect has got adaptive adjustment. In particular, an adaptive partition algorithm is proposed to reduce computation time. The performance of the method is examined numerically and experimentally, which is compared with the other methods. The results provide the method to guide the design of a 64-microphone optimized array with high performance ( $1.8^{\circ}$ spatial angle resolution and $40 \%$ Doppler frequency correction over the bandwidth from $800 \mathrm{~Hz}$ to $3000 \mathrm{~Hz}$ ) and fast computing speed (18 s array generated time for 2000 arrays). Furthermore, an unusual feature of the method is that it can be utilized in the case when the source moves at a nonconstant velocity.

\section{Introduction}

Acoustic array techniques are applied for moving sources, such as cars [1], trains [2], airplanes [3-5], and wind turbines [6-8]. However, array identifying accuracy and resolution are limited by the array configuration due to complex motion sound characteristics. Moving sound sources need some special acoustic characteristics such as broadband, Doppler effect, and directivity, which undoubtedly impose high requirements on array configuration design. For instance, in this case, the array microphones must be mounted closer to avoid spatial aliasing. Also, the total array aperture must be large enough to allow moving sources to be solved accurately. Unfortunately, although the traditional regular array [9] had much more advantages on array performance, it is helpless to meet these requirements due to need for a large number of microphones. Fortunately, irregular array $[10,11]$ is a good method by which the larger array aperture can be obtained conveniently with few microphones. Irregular array consists of two major categories including geometrical and performance optimized arrays. Ignoring the fact that further studies are required to clarify kinds of optimized arrays, a detailed explanation of the two-dimensional geometrical array is provided in this paper. Geometrical arrays are subdivided into sparse array [12, 13], random array [14], and random sparse array [15]. Sparse arrays are generated from the regular array by extracting different spacing microphones. It provides the relatively larger apertures with the same number of microphones. However, a sparse array is also redundant arrays, which is just like a regular array. Severe aliasing problem always occurs because of the repeated sampling spacing. Although this phenomenon can be improved by limiting the minimum intrasensor spacing less than half wavelength, spatial aliasing still results in the resolution degradation and the appearance of ghost sources in acoustic map [16]. In fact, in order to significantly reduce spatial aliasing, microphone 
arrays must guarantee nonredundancy in spatial sampling, i.e., nonredundant with almost unique intramicrophone spacings.

Array microphones mounted randomly are one strategy to ensure nonredundant array generation. The position of microphones in a random fashion prevents the periodicity inherent in nonrandom arrangements [17]. However, this randomly layout mode easily leads to the microphones clumping together in a small region, thus yielding a smaller effective aperture size. This problem has been addressed by employing segmenting scheme [18]. Furthermore, earlier studies have shown that the geometrical rotation symmetry helps to avoid array redundancy, for example, wheel array with the odd number of spokes [19] and pizza array with odd slices [20]. Previous research has found that uniform density of the microphone distribution was a significant geometrical factor. Because different points on the source plane will have different exposure from the array, it affects array effective measurement especially irregular array. To ensure the effectiveness, Hook and coworkers [18] have handled this problem of a regular grid array by employing uniform segment scheme. The work described in the present article was performed to evaluate the uniformity for an irregular array.

Despite there is considerable research studies, a good irregular array design is still difficult to conduct due to unexplained relationship among array geometry, performance, and incomplete performance indicators. Some research studies [21] attempted to make it clear what geometric properties are crucial for the superior beamforming performance of irregular arrays. Others [22] wanted to explain that how array layout affect the performance by random, genetic, and iterative optimization. However, because optimization procedures are an exhaustive search, it often resorts to a tedious trial and error cycle to design irregular arrays for a given frequency range. Moreover, since the trade-off of array geometry and performance always heavily relies on situations of application, there are no consistent standards and principles to follow. Since sidelobe of irregular array beampattern is more complex than that of regular configurations on the aspect of amplitude and shape, performance indicators are more intricate problem. So far, a lot of research studies have been conducted to enhance array performance by employing maximum sidelobe level (MSL) [22], synthesizing mainlobe width (MW) and MSL [23], adopting product of frequency-averaged MLW and MSL [24, 25], using statistical characteristics of MW and MSL [18], and utilizing the relative variances of MW and MSL [26-28]. Although those methods have the ability to cover large apertures with quite few microphones, low MSL in a wide range of frequencies, and good geometry symmetry, there are some challenges in designing the high performance irregular array for the localization of moving sources. Firstly, the effective geometrical parameters and performance indicators are needed, which can lead to a quantifying relationship between array geometry and performance. Secondly, a more effective cost function can manage the spatial variation of array beampattern. Finally, a simple and fast optimization algorithm is needed for improving computation speed.

In this paper, a partition spatial filtering method is put forward to deal with the problem of irregular array configuration. A geometrical parameter (evenness index) and performance indicator (sidelobe suppression ratio) are established by introducing irregular grids spacing and partition filtering conditions. Our intention is to describe clearly the relationship between irregular array geometry and performance. In particular, an adaptive partition algorithm is designed to enhance optimized speed. Compared with the popular irregular arrays such as logical spiral wheel array and pizza array, partition optimized array has shown superior array performance especially resolution and corrected ability of Doppler effect. The method is more suitable for designing irregular array to localize moving wideband sources.

\section{Irregular Grid Array for Moving Sources}

A standard moving source array measurement is described in Figure 1, in which the height from the array to the ground is $h$ and the focused distance is $Z_{0}$. The source locations are assumed on a reconstruction plane $\mathrm{B}$ which is attaching to and moving with the side plane of the moving sources. Since the source position can be regarded as a function of time, propagation distances from assumed source locations to each array microphone in the array plane A are calculated. Based on propagation distances, microphone signals are deDopplerized corrected. The processed signals are focused on a sequence of locations on the reconstruction plane $B$ to yield the spatial distribution of source strength and then to realize source identification.

During the measurement of moving sources, an array must satisfy basic requirements that there are at least two microphone spacings within one spatial cycle of the sound field in the $x$ direction. Therefore, to avoid spatial aliasing, the minimum sensor spacing is allowed in the $x$ direction, which can be calculated as

$$
d_{\min }=c \cdot\left[2 f_{\max } \sin (\theta)\right]^{-1},
$$

where $c$ is the sound speed and $f_{\max }$ is the highest frequency that contributes to the sound field incident on the array. The present analysis is built on the existence of plane phase planes. An assumed spherical phase surfaces would result in a decrease in the allowable microphone spacing in the $x$ direction.

2.1. Irregular Grid Spacing. In order to share the same spatial aliasing characteristics and avoid the intrinsic periodicity of regular layout, an irregular array is determined on the irregular grid. Irregular grid is built on coaxial ring array which is circumferential equal interval and radial nonuniformly spacing. The spacings of irregular grid are utilized to control the smallest actual microphone spacing for suppressing spatial aliasing. The unequal radius is derived from the performance synthesis of a uniform coaxial ring 


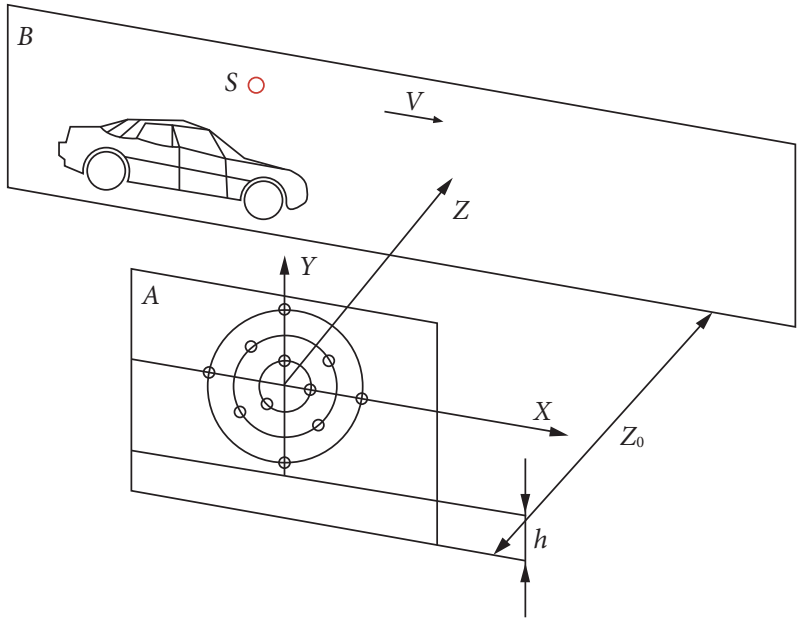

(a)

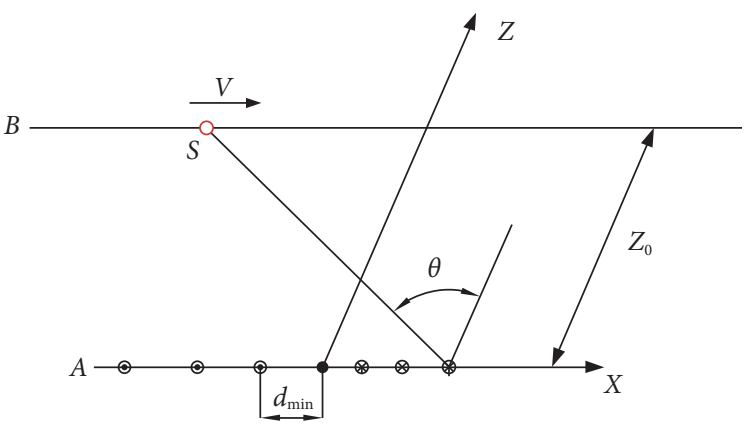

(b)

Figure 1: The relationship between the measurement array and reconstruction field: (a) isometric view; (b) top view.

array, which is shown in Figure 2. Coaxial ring array maintains the rotational symmetry as the wheel array and pizza array. It benefits for avoiding redundant spatial twodimensional sampling of the array. Moreover, based on the geometric symmetry, it is easily amplifying array aperture through increasing the number of rings or ring spacing, and the array aperture is increasing with twice the radius length. Therefore, an irregular coaxial ring array is generated, and its irregular grid spacings are determined.

First of all, circumferential direction grids are segmented evenly with the equal arc length interval $d$ which should satisfy $0.25 \lambda \leq d \leq 0.5 \lambda$ to ensure that the array can reduce mutual coupling effects in the upper frequency limit. The normalized $d$ by a wavelength $\lambda$ is expressed as $d$ $(0.25 \leq \widetilde{d} \leq 0.5)$. Furthermore, the maximum ring radius $\rho_{\max }$ equals to the aperture, and the adjacent ring radius difference $\Delta \rho$ fits to $0.5 \lambda \leq \Delta \rho \leq \lambda$. The normalized $\Delta \rho$ by wavelength $\lambda$ is expressed as $\Delta \widetilde{\rho}(0.5 \leq \Delta \widetilde{\rho}<1)$. The minimized microphone spacing is effectively guaranteed by controlling the radius of the inner ring. For moving source measure, the innermost ring radius $\rho_{1}$ is determined by $\rho_{1}=c \cdot\left(2 f_{\max }\right)^{-1}$. Secondly, based on the uniform coaxial ring array, the unequal rings' radius can be deduced by the sinusoidal transformation of a regular coaxial ring array's pattern. Uniform coaxial ring array beampattern is given as

$$
E(\theta, \phi)=\sum_{i=1}^{N} \sum_{j=1}^{M_{i}} P_{i j} e^{j k \rho_{i} \sin (\theta) \cos \left(\phi-\phi_{j}\right)},
$$

where $N$ is the number of coaxial rings, $M_{i}$ is the number of elements in the $i$ th ring, $P_{i j}$ is the sound pressure response of the element $j$ in ring $i$, and $\rho_{i}$ is the radial distance to the $i$ th ring from the center of the array. $k\left(k=2 \pi \cdot \lambda^{-1}\right)$ is the wave number. For simplification of deducing, the sound pressure response is assumed to be uniform, i.e., $P_{i j}=1$. The elements in each ring are also assumed to be uniformly distributed around the circular ring, i.e., $\phi_{j}=2 \pi j \cdot M_{j}^{-1}$. After considerable manipulation, we can obtain

$$
E(\theta, \phi)=\sum_{i=1}^{N} M_{i} J_{0}\left(k \rho_{i} u\right),
$$

where $u=\sin (\theta)$ and $J_{0}(\cdot)$ is the Bessel function of zero order.

Sinusoidal transformation of equation (3) can be expressed as

$$
F\left(b_{p}\right)=\sum_{i=1}^{N} M_{i} \int_{0}^{\pi} J_{0}\left(k \rho_{i} u\right) \sin \left(b_{p} u\right) \mathrm{d} u .
$$

Assuming that $f\left(a_{i}, b_{p}\right)=\int_{0}^{\pi} J_{0}\left(a_{i} u\right) \sin \left(b_{p} u\right) \mathrm{d} u$ and using the properties of $J_{0}$, we can obtain

$$
f\left(a_{i}, b_{p}\right)= \begin{cases}\left(a_{i}^{2}-b_{p}^{2}\right)^{-0.5}, & a_{i}<b_{p}, \\ 0, & b_{p}<a_{i} .\end{cases}
$$

Combination with arranging equations (4) and (5), $a_{p}$ is determined by the auto regression.

$$
a_{p}=\left(b_{p}^{2}-M_{p}^{2} \cdot\left[F\left(b_{p}\right)-\sum_{i=1}^{p-1} M_{i} f\left(b_{p}, a_{i}\right)\right]^{-2}\right)^{0.5} .
$$

pth ring radius is also obtained by

$$
\rho_{p}=a_{p} \cdot k^{-1} .
$$

Finally, once the circumferential spacing and rings' radius are defined, the underlying irregular grid points can be generated, which is shown in Figure 2. In the figure, the black dots depict grid points and each grid point is a potential position of the array microphone.

2.2. Irregular Grid Point. In this section, the number of grid point on each ring $M_{i}(i=1,2, \ldots, N)$, the total number of grid point $M_{t}$ with given ring's number $N$, and the quantitative relationship between grid point increment $\Delta m$ and ring radius increment $\Delta \widetilde{\rho}$ are derived. 


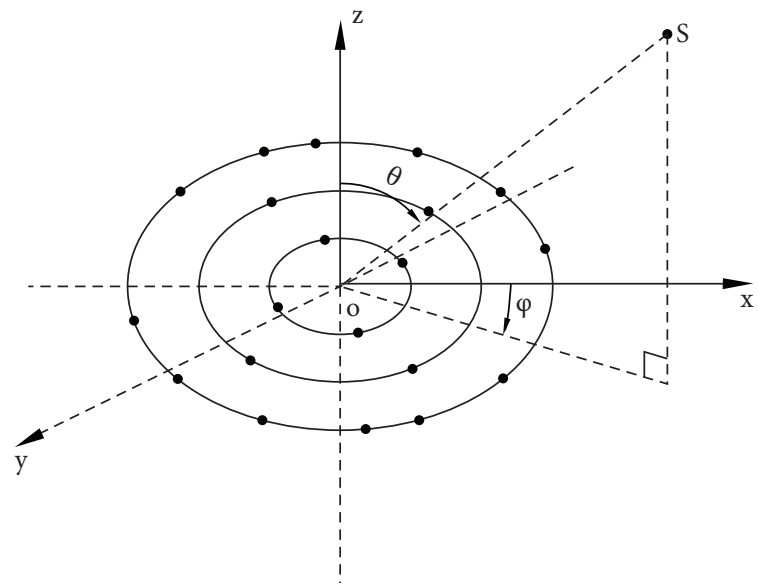

(a)

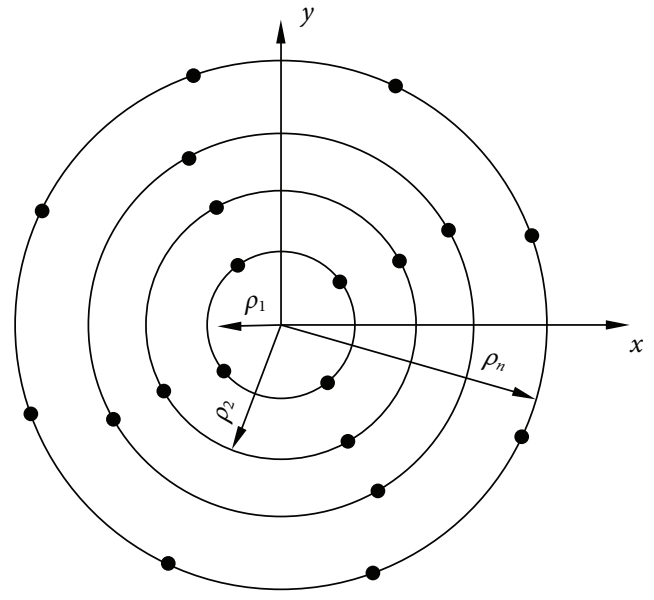

(b)

Figure 2: Geometry of a coaxial ring array and grid points: (a) isometric view; (b) top view.

The arc length interval $d_{i}$ and the ring radius $\rho_{i}$ are normalized by wavelength $\lambda$. They are defined as

$$
\begin{gathered}
\tilde{d}_{i}=2 \pi \rho_{i}\left(M_{i} \lambda\right)^{-1}, \\
\tilde{\rho}_{i}=\tilde{d}_{i} M_{i} \cdot(2 \pi)^{-1} .
\end{gathered}
$$

The difference of the grid point's number in adjacent rings is named grid point increment $\Delta m$ which is defined by

$$
\Delta m=M_{i+1}-M_{i} .
$$

Normalized difference of radius in adjacent rings is called radius increment $\Delta \widetilde{\rho}$ which is defined as

$$
\Delta \widetilde{\rho}=\tilde{\rho}_{i+1}-\tilde{\rho}_{i} .
$$

Assuming the arc length intervals of all rings are consistent, i.e., $\widetilde{d}_{1}=\widetilde{d}_{2}=\cdots=\widetilde{d}_{N}=\widetilde{d}$, we can obtain the quantitative relationship between grid point increment $\Delta m$ and ring radius increment $\Delta \widetilde{\rho}$, where

$$
\Delta m=2 \pi \Delta \tilde{\rho} \cdot \tilde{d}^{-1} .
$$

In the case of $0.25 \leq \tilde{d} \leq 0.5$, grid point increment $\Delta m$ can be depicted as

$$
\operatorname{int}(2 \pi) \leq \Delta m \leq \operatorname{int}(8 \pi),
$$

where int expresses getting an integer.

The total grid point's number $M_{t}$ of a coaxial rings array with $N$ rings can be expressed as

$$
M_{t}=N M_{1}+\sum_{i=1}^{N-1} i \Delta m_{i}
$$

where $\Delta m_{i}$ is the grid point's increment of the ring $i$ and $M_{1}$ is the grid point number of the inner ring. The concrete relations among $\Delta m, \Delta \widetilde{\rho}$, and $\widetilde{d}$ are plotted in Figure 3 .

\section{Adaptive Partition Filtering}

The performance of an irregular array, the mainlobe width, and the peak sidelobe level depends on the aperture size and the way of the microphones disposed on the grid points. To make sure that the microphones are spatial well distributed after fixed an appropriate grid size, the entire aperture into smaller subsections was considered. Also, quantitative array spatial filter conditions and performance evaluating indicators were proposed in the paper.

In the partition procedure, microphones are first grouped on a segment-by-segment basis, and each group of microphones is positioned randomly on the grid points within each subsection. Taking into account the measurement of a wide frequency band, one microphone is always positioned at the center of the array's aperture, named zero section, and the rest of the microphones were positioned randomly within the various subsections. There is equal number of microphone and grid point in each subsection. The microphones in each subsection is placed at least four, i.e., the microphone number in the outmost ring is the same as the subsection number $C$. The subsection number is strongly related to array microphones' number $M_{C}$, grid points' number $M_{t}$, circular rings' number $N$, and incident wave length. A novel adaptive algorithm is introduced to describe this relation.

In order to guarantee the uniformity of grid points' distribution, the ratio between microphones' number $M_{s}\left(M_{s}=M_{C} \cdot C^{-1}\right)$ and grid points' number $J\left(J=M_{t}\right.$. $\mathrm{C}^{-1}$ ) in each subsection is defined as

$$
M_{s} \cdot J^{-1}=M_{C} \cdot M_{t}^{-1} \geq 6 .
$$

Another constraint is imposed on the grid points in each ring to ensure the large array aperture and wide range of measurement frequency, which is defined by 


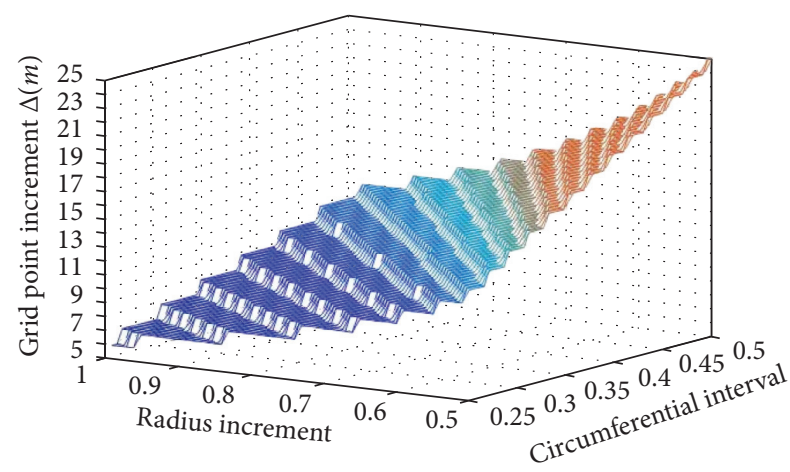

FIGURE 3: Relations among grid point increment $\Delta m$, radius increment $\Delta \widetilde{\rho}$, and circumferential interval $\widetilde{d}$.

$$
\left\{\begin{array}{l}
M_{i} \geq \operatorname{int}\left(C \cdot J^{-1}\right) \times i \\
Q_{i}=\operatorname{int}\left(C \cdot J^{-1}\right) \times i
\end{array}\right.
$$

where $Q_{i}$ is the number of polar angle in the $i$ th ring in each subsection.

Once given the subsections' number $C$ and grid points' number $J$ in each subsection, the mathematical model of array's grid points is generated. Based on the geometric symmetry, the coaxial ring array can be transformed into the matrix expression of a polar diameter and the polar angle of the polar coordinate system. A grid point can be described by a pair of polar angle and polar radius. The grid points of the entire underlying coaxial ring array are depicted by the polar angles' matrix and polar radius's matrix. The mathematical model is described as

$$
\begin{aligned}
\mathbf{R} & =\left(\begin{array}{cccc}
\rho_{1} & \rho_{1} & \cdots & \rho_{1} \\
\rho_{2} & \rho_{2} & \cdots & \rho_{2} \\
\vdots & \vdots & \ddots & \vdots \\
\rho_{J} & \rho_{J} & \cdots & \rho_{J}
\end{array}\right)_{J \times C}, \\
\boldsymbol{\Phi} & =\left(\begin{array}{cccc}
\varphi_{11} & \varphi_{12} & \cdots & \varphi_{1 C} \\
\varphi_{21} & \varphi_{22} & \cdots & \varphi_{2 C} \\
\vdots & \vdots & \ddots & \vdots \\
\varphi_{J 1} & \varphi_{J 2} & \cdots & \varphi_{J C}
\end{array}\right)_{J \times C} .
\end{aligned}
$$

The columns of matrix $\mathbf{R}$ and $\Phi$ depict the subsections, and their rows represent the polar radius and polar angle of grid points over array area, respectively. The polar angle is named subsection angle which starts on one side of the subsection's boundary line, not in the positive direction of the $x$-axis of the array. They are distributed in each subsection randomly, and all the angles are not equal.

An irregular array can be generated using the polar radius $R^{\prime}$ and polar angle $\Phi^{\prime}$ matrixes, which are generated by positioning randomly several nonzero elements in each column of matrices $\mathbf{R}$ and $\Phi$ in equations (16) and (17). The polar radius $R^{\prime}$ and polar angle $\Phi^{\prime}$ matrixes are shown in the following equations:

$$
\begin{aligned}
\mathbf{R}^{\prime} & =\left(\begin{array}{cccc}
\rho_{1} & \rho_{1} & \cdots & \rho_{1} \\
\rho_{2} & \rho_{2} & \cdots & 0 \\
0 & \rho_{3} & \cdots & \rho_{3} \\
\vdots & \vdots & \ddots & \vdots \\
\rho_{J-1} & 0 & \cdots & \rho_{J-1} \\
\rho_{J} & \rho_{J} & \cdots & \rho_{J}
\end{array}\right)_{J \times C}, \\
\boldsymbol{\Phi}^{\prime} & =\left(\begin{array}{cccc}
\varphi_{11} & \varphi_{12} & \cdots & \varphi_{1 C} \\
\varphi_{21} & \varphi_{22} & \cdots & \varphi_{2 C} \\
0 & \varphi_{32} & \cdots & 0 \\
\vdots & \vdots & \ddots & \vdots \\
\varphi_{J-11} & 0 & 0 & \varphi_{J-1 C} \\
\varphi_{J 1} & \varphi_{J 2} & \cdots & \varphi_{J C}
\end{array}\right)_{J \times C} .
\end{aligned}
$$

The above matrix operations will produce a mass of polar radius and polar angle matrices, i.e., plentiful array configurations. It resorts to a tedious trial and error cycle. In order to overcome this problem, some novel spatial filter conditions are proposed to filter out array configurations having relatively larger mainlobe width for the same sidelobe level.

3.1. Filtering Condition. There are two aspects need to be considered for imposing geometric constraints on the configuration for array preselecting. Firstly, since the regularity of microphone position leads to redundant spatial 2D sampling interval, we employed the microphone position's general angle and circumferential general angle difference in the same ring to avoid regularity. They are given in Condition 1. Secondly, uniform density of microphones across the array area is the main factor in the variation of the measurement distances of moving sources. When the array is used at sufficiently long measurement distance, typically down to the distance equal to array diameter, the nonuniform density ensures good array performance over a wide frequency range. However, if the distance becomes much smaller than that, the nonuniform density of the microphones across the array area starts to have the effect that different points on the source plane will get very diverse exposure from the array. In this case, a more uniform density might be better, and numerical simulations have verified that hypothesis-specifically for the so-called partition array described in the present work. When the same array has to be used at very small measurement distances, a more uniform density is even more necessary. In order to quantitatively evaluate the uniformity density of microphones, a novel geometry descriptor is put forward to capture the properties of microphone distribution showing their impact on array performance. They are shown in Condition 2.

Condition 1. Constraints are imposed on the general angle and circumferential general angle difference.

General angle is an angle of the coordinate with the positive direction of the polar axis as the starting position. It can be defined from the subsection angle as follows: 


$$
\varphi_{k q}^{\prime}=2 \pi \cdot C^{-1} \cdot(q-1)+\varphi_{k q},
$$

where $q$ is the index of subsections number $(q=1,2, \ldots, C)$, $\varphi_{k q}$ is the angle $k$ in subsection $q(k=1,2, \ldots, J)$, and $\varphi_{k q}^{\prime}$ expresses the general angle $k$ in subsection $q$. The array's general angle can be expressed as the form of matrix as follows:

$$
\Phi=\left[\phi_{1}, \phi_{2}, \ldots, \phi_{i}, \ldots, \phi_{N}\right]=\left(\begin{array}{c}
\varphi_{111}^{\prime} \\
\varphi_{221}^{\prime} \\
\vdots \\
\varphi_{M_{1} 41}^{\prime} \\
0 \\
0 \\
0
\end{array}\right.
$$

The column vector $\varphi_{\mathbf{i}}=\left(\varphi_{11 i}^{\prime}, \varphi_{22 i}^{\prime}, \ldots, \varphi_{k q i}^{\prime}, \ldots, \varphi_{M_{i} C i}^{\prime}\right)^{T}$, where $\varphi_{k q i}{ }^{\prime}$ is the $k$ th general angle in ring $i$ and subsection $q$, which expresses the general angle with equal radius. Since the number of microphones in each ring is different, we use zeros to express subsections without microphones in ring $i$, and in case of $M_{i}<M_{\max }$, row zero should be padded in $\varphi_{i}$. Transform the matrix $\Phi$. Firstly, circumferential general angle difference in the same ring for instance, assuming $\varphi_{i}=$ $\left(\varphi_{11 i}^{\prime}, \varphi_{22 i}^{\prime}, 0, \varphi_{34 i}^{\prime}, 0, \varphi_{47 i}^{\prime}, 0\right)^{T}$ to show that seven subsections, four microphones in ring $i$ and microphones are placed in subsections 1, 2, 4, and 7, respectively. The transformation is defined as

$$
\begin{aligned}
\boldsymbol{\Omega} & =\left[\begin{array}{cccc}
0 & \varphi_{11 i}^{\prime}-\varphi_{22 i}^{\prime} & \varphi_{11 i}^{\prime}-\varphi_{34 i}^{\prime} & \varphi_{11 i}^{\prime}-\varphi_{47 i}^{\prime} \\
0 & 0 & \varphi_{22 i}^{\prime}-\varphi_{34 i}^{\prime} & \varphi_{22 i}^{\prime}-\varphi_{34 i}^{\prime} \\
0 & 0 & 0 & \varphi_{34 i}^{\prime}-\varphi_{47 i}^{\prime} \\
0 & 0 & 0 & 0
\end{array}\right] \\
& =\left[\begin{array}{cccc}
0 & \Delta \varphi_{12} & \Delta \varphi_{14} & \Delta \varphi_{17} \\
0 & 0 & \Delta \varphi_{24} & \Delta \varphi_{27} \\
0 & 0 & 0 & \Delta \varphi_{47} \\
0 & 0 & 0 & 0
\end{array}\right],
\end{aligned}
$$

where $\Delta \varphi_{12} \cdots \Delta \varphi_{47}$ express circumferential general angle differences among different subsections in the same ring, i.e., $\Delta \varphi_{12}$ is angle difference between 1 st and 2 nd subsections and other analogies. The elements of angle difference matrix $\Omega$ should satisfy

$$
\psi=\left(\varphi_{1}^{\prime}, \varphi_{2}^{\prime}, \ldots, \varphi_{q}^{\prime}, \ldots, \varphi_{C}^{\prime}\right)
$$

where $\varphi_{\mathbf{q}}^{\prime}=\left(\varphi_{1 q}^{\prime}, \varphi_{2 q}^{\prime}, \ldots, \varphi_{k q}^{\prime}, \ldots, \varphi_{J q}^{\prime}\right)^{T}$ represents the general angle vector of the grid points in subsection $q$.

Recombination matrix $\psi$ obtains a matrix $\Phi$ as follows:

$$
\left.\begin{array}{ccccc}
\varphi_{112}^{\prime} & \cdots & \varphi_{11 i}^{\prime} & \cdots & \varphi_{11 N}^{\prime} \\
\varphi_{222}^{\prime} & \cdots & 0 & \cdots & \varphi_{21 N}^{\prime} \\
\varphi_{332}^{\prime} & \cdots & \varphi_{23 i}^{\prime} & \cdots & \varphi_{31 N}^{\prime} \\
\vdots & \vdots & \vdots & \vdots & \varphi_{42 N}^{\prime} \\
\varphi_{M_{2} 52}^{\prime} & \vdots & \varphi_{k q i}^{\prime} & \vdots & \vdots \\
0 & \cdots & \varphi_{M_{i} C i}^{\prime} & \cdots & 0 \\
0 & \cdots & 0 & \cdots & \varphi_{M_{\max } C N}^{\prime}
\end{array}\right)_{M_{\max } \times N}
$$

$$
\Delta \varphi_{p q} \geq 2 \pi \cdot C^{-1}, \quad(p=1,2, \ldots, C ; q=1,2, \ldots, C) .
$$

Secondly, when the number of microphones in the subsection is less than or equal to the rings' number $N$, i.e., $M_{s} \leq N$, there is only one microphone placed in the same ring and same subsection. For example, assume $\varphi_{q}=\left(\varphi_{1 q 1}^{\prime}, \varphi_{2 q 2}^{\prime}\right.$, $\left.0, \varphi_{3 q 4}^{\prime}, \varphi_{4 q 5}^{\prime}, \varphi_{5 q 6}^{\prime}, 0\right)$ is a row vector of matrix $\Phi$. It shows that there are 7 rings and 5 microphones randomly placed in 1st, $2 \mathrm{nd}, 4$ th, 5th, and 6 th rings in subsection $q$. The transformation is defined by

$$
\begin{aligned}
\boldsymbol{\Omega}^{\prime} & =\left[\begin{array}{cccc}
\varphi_{1 q 1}^{\prime}-\varphi_{2 q 2}^{\prime} & \varphi_{1 q 1}^{\prime}-\varphi_{3 q 4}^{\prime} & \varphi_{1 q 1}^{\prime}-\varphi_{4 q 5}^{\prime} & \varphi_{1 q 1}^{\prime}-\varphi_{5 q 6}^{\prime} \\
0 & \varphi_{2 q 2}^{\prime}-\varphi_{3 q 4}^{\prime} & \varphi_{2 q 2}^{\prime}-\varphi_{4 q 5}^{\prime} & \varphi_{2 q 2}^{\prime}-\varphi_{5 q 6}^{\prime} \\
0 & 0 & \varphi_{3 q 4}^{\prime}-\varphi_{4 q 5}^{\prime} & \varphi_{3 q 4}^{\prime}-\varphi_{5 q 6}^{\prime} \\
0 & 0 & 0 & \varphi_{4 q 5}^{\prime}-\varphi_{5 q 6}^{\prime}
\end{array}\right] \\
& =\left[\begin{array}{cccc}
\Delta \varphi_{12}^{\prime} & \Delta \varphi_{14}^{\prime} & \Delta \varphi_{15}^{\prime} & \Delta \varphi_{16}^{\prime} \\
0 & \Delta \varphi_{24}^{\prime} & \Delta \varphi_{25}^{\prime} & \Delta \varphi_{26}^{\prime} \\
0 & 0 & \Delta \varphi_{45}^{\prime} & \Delta \varphi_{46}^{\prime} \\
0 & 0 & 0 & \Delta \varphi_{56}^{\prime}
\end{array}\right],
\end{aligned}
$$

where $\Delta \varphi_{12}^{\prime} \cdots \Delta \varphi_{56}^{\prime}$ express angle differences among different rings in the same subsection, i.e., $\Delta \varphi_{12}^{\prime}$ is angle difference between 1 st and 2 nd rings and other analogies. The elements of angle difference matrix $\Omega^{\prime}$ should satisfy

$$
\left\{\begin{array}{ll}
\Delta \varphi_{p^{\prime}, q^{\prime}}^{\prime} \neq 2 \pi \cdot(J C)^{-1}, & \left(p^{\prime}=q^{\prime}\right), \\
\Delta \varphi_{p^{\prime}, q^{\prime}}^{\prime} \geq 2 \tilde{d} \pi \cdot(180 \Delta \widetilde{\rho})^{-1}+2 \pi\left(p^{\prime}-q^{\prime}\right) \cdot(J C)^{-1}, & \left(p^{\prime} \neq q^{\prime}\right),
\end{array} \quad\left(\begin{array}{l}
p^{\prime}=1,2, \ldots, M_{s} \\
q^{\prime}=1,2, \ldots, M_{s}
\end{array}\right) .\right.
$$


Furthermore, in the case of $M_{s}>N$, i.e., the number of microphone $M_{s}$ is more than the rings' number $N$ in the same subsection. This means that there are at least two microphones in the same ring and same subsection. The general circumferential angle difference of adjacent microphones in the same ring should abide by the condition of equation (26) $\left(p^{\prime}=q^{\prime}\right)$.

Condition 2. Geometric descriptor assesses uniformity of microphone's distribution. The mass center of array is denoted as

$$
\left(x_{0}, y_{0}\right)=\left(M_{c}^{-1} \cdot \sum_{i=1}^{M_{c}} x_{i}, M_{c}^{-1} \cdot \sum_{i=1}^{M_{c}} y_{i}\right),
$$

where $x_{i}=\rho_{i} \cos \left(\phi_{i}\right)$ and $y_{i}=\rho_{i} \sin \left(\phi_{i}\right)$ express the $i$ th microphone position. Its polar moment is defined as

$$
L_{i}=\left[\left(x_{i}-x_{0}\right)^{2}+\left(y_{i}-y_{0}\right)^{2}\right] \text {. }
$$

Based on equation (28), a definition of aperture size for irregular array can be achieved. The aperture can be regarded as approximately proportional to the dispersion of the microphones. The dispersion factor used to evaluate microphone dispersion is presented. It is defined that the average microphone polar moment is computed by

$$
a=M_{c}^{-1} \cdot \sum_{i=1}^{M_{c}}\left|L_{i}\right|
$$

Furthermore, Pielou's evenness index EI, which is normalized Shannon entropy, is introduced to numerically assess the uniformity of microphone distribution.

$$
\mathrm{EI}=H \cdot H_{\max }^{-1}=-\sum_{i=1}^{M_{c}} g_{i} \operatorname{Ln}\left(g_{i}\right) \cdot[\operatorname{Ln}(s)]^{-1},
$$

where $s$ is the total number of $L_{i}, g_{i}$ is the percentage of $L_{i}$ within $a, H$ is the Shannon entropy, and $H_{\max }$ is the maximum possible entropy which represents an ideal uniform distribution of $L_{i}$. As the value of EI increases, microphones tend to spread evenly over the array area.

3.2. Performance Evaluating Indicator. The main parameters influencing the performance of an array are the mainlobe width (MW) and sidelobe level. Sidelobe level consists of the maximum sidelobe level (MSL) and sidelobe suppression ratio (SSR). All parameters present here are on the basis of the so-called array pattern. The mainlobe width represents the spatial resolution of the array and the ability of the array to distinguish different sound sources. The sidelobe level represents the performance of the array in suppressing nonsonic or false sources. The narrower mainlobe width and the smaller sidelobe level, the better the performance of the array.

3.2.1. Mainlobe Width. Mainlobe width represents array's spatial resolution, that is to say, the ability of the array to distinguish different sound sources. It is a common parameter to assess the resolution of array. The narrower the mainlobe width is, the higher the resolution has. The mainlobe width, in particular, is strongly related to an array configuration. There are different definitions of mainlobe width. We adopt $3 \mathrm{~dB}$ down mainlobe width in this paper.

It is known that an array's mainlobe width generally depends on the array's aperture size. Aperture sizes of irregular array to some extent can be controlled by the size of the underlying coaxial rings. Here is a definition of "aperture size" for two-dimensional arrays. It can be expressed as the average dispersity of the polar moment of an array's microphone positions on the array area. The diffusivity $a$ is determined by equation (29). In the simulation section, the relationship between the mainlobe width and aperture size is investigated based on statistical analysis of arrays generated for different segmenting schemes.

3.2.2. Sidelobe Level. The sidelobe level is related to the noise rejection capability of an array. And, it is usually an important factor of the performance of noise. Maximum sidelobe level (MSL) is one of evaluative indicators for the sidelobe level. It defines the dynamic range of the ability of the array to separate sources in different directions. The MSL is a significant parameter when choosing an optimal array configuration. On the contrary, when interpreting results obtained by a given array, it is important to know about MSL. Therefore, a good array design can be characterized by having lower MSL, measured relative to the mainlobe level. Based on the profile of the array pattern shown in Figure 4, we define the maximum sidelobe level function as

$$
\mathrm{MSL}=10 \log _{10}\left(h_{v}^{-1}\right) .
$$

It is known that the peak sidelobe levels of irregular arrays tend to be higher than those of equivalent fully populated arrays, and the shape of sidelobe profile is more complex than it. In order to comprehensively assess the performance for an array configuration, sidelobe suppression ratio (SSR) is presented. It is defined as

$$
r_{\text {sp }}=20 \log _{10}\left(h_{p} \cdot h_{v}^{-1}\right) \text {, }
$$

where $h_{v}$ and $h_{p}$ are the amplitude of maximum sidelobe peak and the amplitude of mainlobe peak, respectively.

The numerical simulation results show that the irregular arrays' sidelobe suppression ratio is strongly related to the number of array microphones. The quantitative relationship can be described as

$$
\begin{cases}r_{\mathrm{sp}} \geq M_{c}-1, & M_{c} \leq 16, \\ r_{\mathrm{sp}} \geq 16, & M_{c}>16,\end{cases}
$$

and this value increases as the number of microphones increases.

\section{Simulations}

The adaptive partition algorithm convergence depends on the irregular grid size, the geometric conditions, and the performance cost functions. The way to verify the ability of 


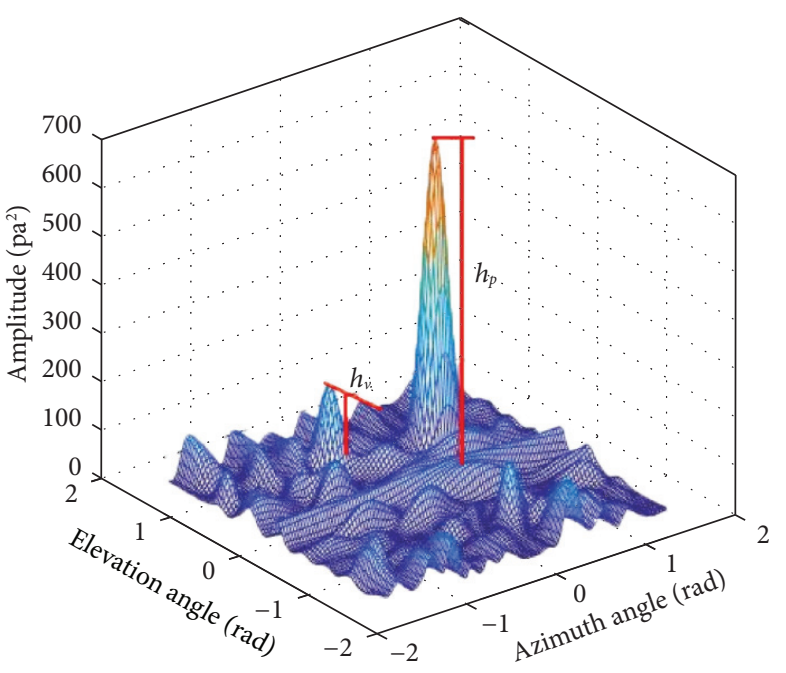

Figure 4: Three dimensional array pattern profile.

the algorithm to provide optimized and repeatable solutions to the selected parameters is to investigate the statistics of various optimized solutions. The performance repeatability is investigated for 2000 arrays optimized in MW, MSL, and SSR, with two types of partition schemes. The objective is to validate the optimization process and also to identify trends. The parameters used for this simulation are shown in Table 1.

First of all, the focus is done on the performance of the partition arrays in terms of EI, MW, MSL, and SSR with different partition schemes. And then, the optimal partition array configuration is investigated and determined. Finally, frequency sensitivity, the resolution, and Doppler effect correction capability of optimal partition array are studied.

4.1. Partition Array Generation. As optimized arrays produce some repeatability in their performance, the purpose is to discern preferential positioning for microphones, which is associated with the given performance indicators that need to be optimized by the adaptive partition optimization algorithm. In the case of the number of microphones in each subsection is more than four, i.e., $M_{s} \geq 4$, 64-microphone array configurations with different partition scheme are plotted in Figure 5. In Figure 5, all outmost radiuses of configurations are the same and the "o" highlights the position of microphones. The values of EI, MW, MSL, and SSR that are taken from 2000 optimized arrays in each partition scheme are plotted in Figure 6.

The best choice of an array configuration from amongst all the partition arrays is reproduced below. Firstly, the array partition is conducted by adaptive partition. Odd number segments include subsections 7 and 9 with a center microphone, which are shown in Figures 5(a) and 5(b). Even number segments consisting of subsections 8 and 16 have no center microphone, which are plotted in Figures 5(c) and 5(d), respectively. And then, candidate arrays are produced by preselecting from different partition arrays using geometrical filter conditions. If an array configuration of (17) does not meet Conditions 1 and 2, the polar radiuses' and
TABLE 1: Simulation parameters.

\begin{tabular}{lc}
\hline Parameters & Values \\
\hline Source azimuth angle $\left(^{\circ}\right)$ & 45 \\
Source elevation angle $\left(^{\circ}\right)$ & $60-120$ \\
Sound pressure level $(\mathrm{dB})$ & $65-75$ \\
Frequency $(\mathrm{Hz})$ & $800-3000$ \\
Movement speed $\left(\mathrm{km} \cdot \mathrm{h}^{-1}\right)$ & $60-120$ \\
Aperture size & $2 \mathrm{~m}\left(5 \lambda_{\max }\right)$ \\
Microphones & 64 \\
Circular rings & 10 \\
\hline
\end{tabular}

polar angles' matrices must be randomly generated again until figure out those matrices of arrays satisfying conditions. Those arrays are called candidate arrays. This procedure is conducted by Matlab random generation. Evenness index EI simulation results are shown Figure 6. Although the introduction of geometrical filter conditions in the generation of partition arrays helps to filter out configurations without fine structural characteristics, the number of candidate arrays is large. And, it is difficult to decide which array is the best. Therefore, to find out the optimal partition array configuration, we need to conduct further assessment by using the performance of the candidate arrays.

Finally, performance indicators have been predefined in Section 3, such as MW, MSL, and SSR. Here, a different compromise principle is set up. It sounds like an appropriate choice of an array configuration is from those arrays having the smallest possible value of MW with the same SSR and the largest EI. Distribution of MW and SSR values of four different partition schemes is plotted in Figures 6(a)-6(d). First of all, the range of MW values becomes narrow as the partition number increases from 7.2, 5.4 (see Figures 6(a)$6(\mathrm{c})$ ) to $5.4^{\circ}$ only (see Figure 6(d)). Moreover, in the same values range, the percentage of the smaller value of $\mathrm{MW}$ increases with the increase in number of partitions; the percentage of 5.4 degree is from $60.0 \%$ (Figure $6(\mathrm{a})$ ) up to $90 \%$ (Figure 6(c)). This is explained by the fact that the partition schemes can enlarge the array's effective aperture. That means as the number of uniform subsections increases, microphones tend to spread evenly over the grid. This is consistent with our original assumption.

On the other hand, range of SSR values becomes narrower and the maximum value is less. The percentage of the value of overtaking 20 drops from $48.5 \%$ (Figure 6(a)) to $12.5 \%$ (Figure 6(d)). This indicates that the increase in the sidelobe level is at the expense of widening MW. This tendency is more obvious in odd number segment schemes because the center microphone is used (Figures 6 6(a) and 6(b)) to enhance the interference suppression. Furthermore, as can be seen from Figures 6(a)-6(d), Pielou's evenness index EI is relative stabilization and the variety is smaller. The mean value is from 3.2 to 2.4 , and the variance is only 0.5 (Figures 6(a) and 6(d)). The value of EI decreases with the increase in partition number approximately. The values of EI of odd number partitions are bigger than those of even number segments. The maximum value of EI is obtained when the partition number is seven. This shows that the seven partition scheme overtakes others schemes on the 


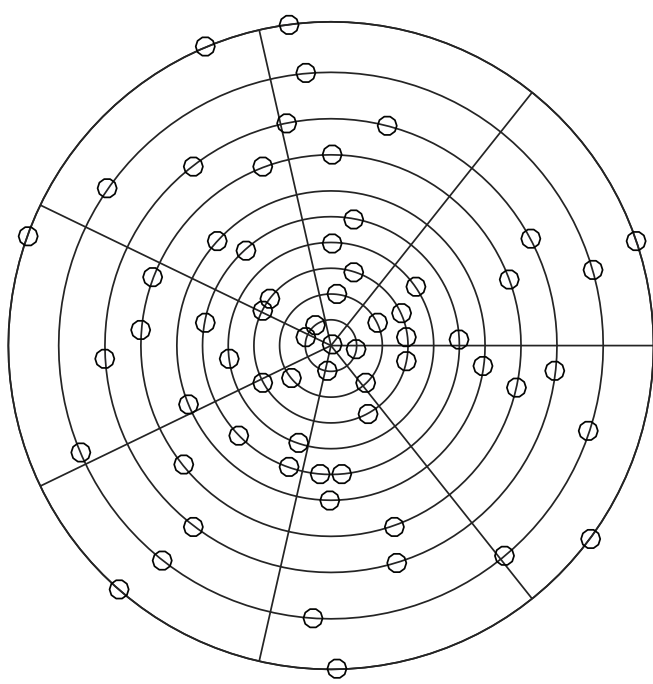

(a)

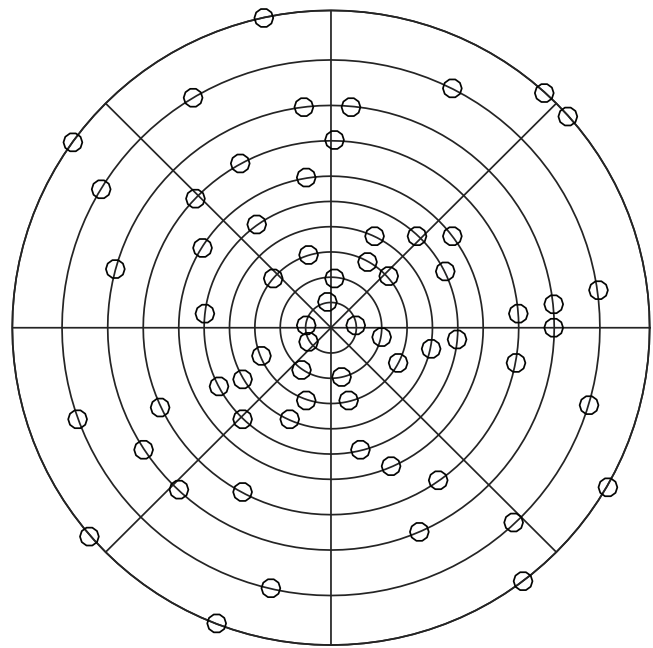

(c)

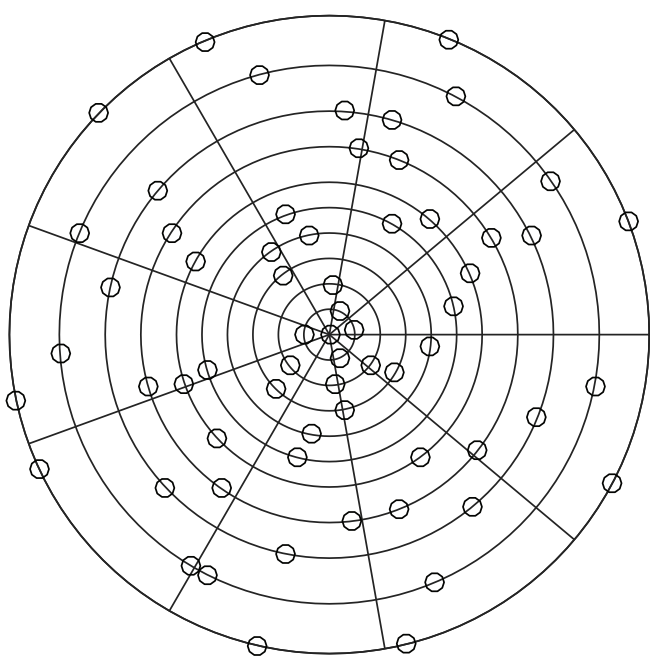

(b)

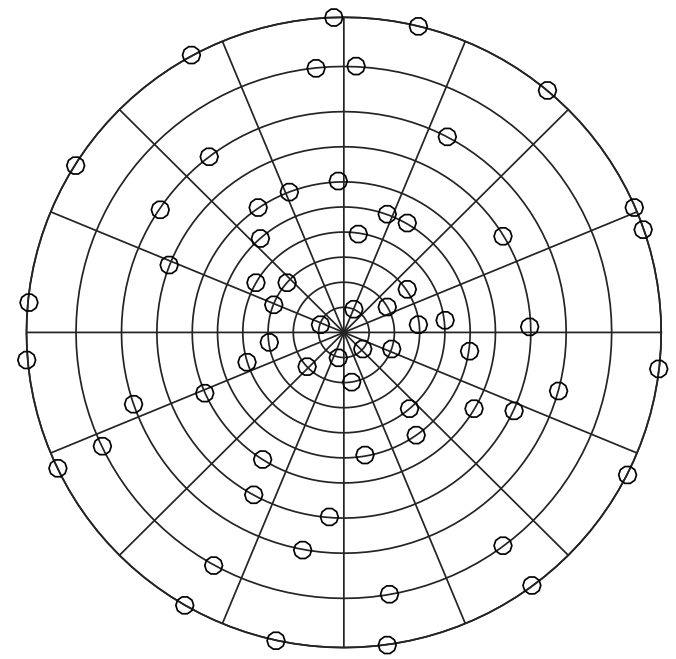

(d)

Figure 5: Partition configurations of 64-microphone array with different of subsection number $C$. (a) $C=7$ and (b) $C=9$ are with central microphone; (c) $C=8$ and (d) $C=16$ are without center microphone.

aspect of the uniformity of microphones distribution. Therefore, the seven partition scheme is our focus. An optimal partition array configuration is generated from above analysis results. It is plotted in Figure 7(c), and its characteristic parameters are given in Table 2.

4.2. Frequency Sensitivity. As arrays are designed for a frequency bandwidth, the evolution of performance indicators with frequency is of great interest to the geometry. Maximum sidelobe level (MSL) and sidelobe suppression ratio (SSR) are two predefined performance indicators which are functions of frequency and directly related to the frequency characteristics of the moving sound sources. A partition array is optimized over the frequency range of $500 \mathrm{~Hz}$ to $3000 \mathrm{~Hz}$, which is shown in Figure 7(c). The same tests are performed using the wheel array and the pizza array to compare the performance, which are shown in
Figures 7(a) and 7(b), respectively. The MSL and SSR curve of three different optimized arrays is calculated, which is shown in Figure 8. These arrays are composed of $64 \mathrm{mi}-$ crophones with $2 \mathrm{~m}$ apertures. Simulation geometrical parameters in our experiment are listed in Tables 1 and 2.

Distributions of SSR and MSL of three different arrays are plotted in Figures 8(a) and 8(b), respectively. As can be seen from Figure 8(a), three arrays have SSR value stability in the frequency range of $800 \mathrm{~Hz}$ to $3000 \mathrm{~Hz}$. Partition array has the maximum SSR value among three arrays. As shown in Figure $8(\mathrm{~b})$, MSL values of partition array are greater than $-10 \mathrm{~dB}$ on the bandwidth of $800 \mathrm{~Hz}$ to $3000 \mathrm{~Hz}$, especially above $1000 \mathrm{~Hz}$ reaching $-8 \mathrm{~dB}$. According to Christensen and Hald [19], a MSL above $10 \mathrm{~dB}$ is a good performance. Therefore, we can obtain that partition array has good false sound source rejection and robustness on the overall radiation bandwidth of moving sound sources, and its frequency performance is superior to log-spiral wheel array and pizza array. 


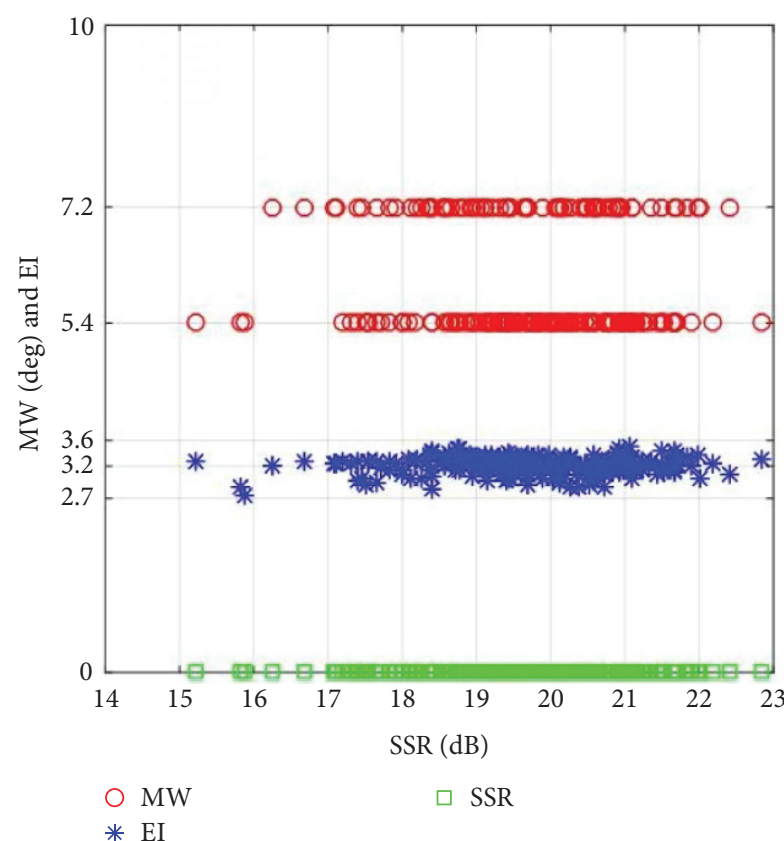

(a)

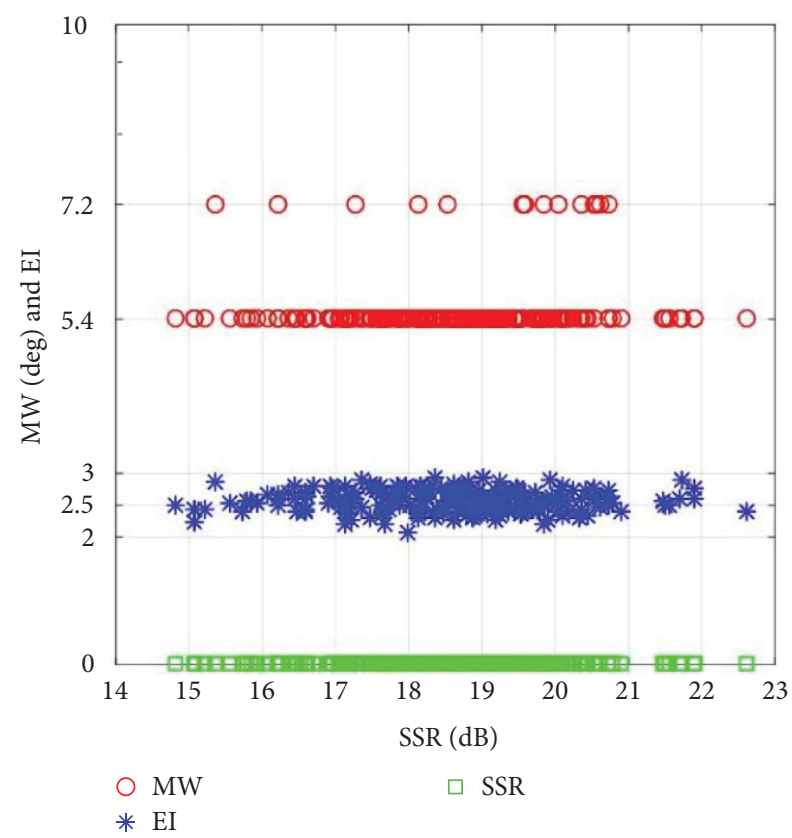

(c)

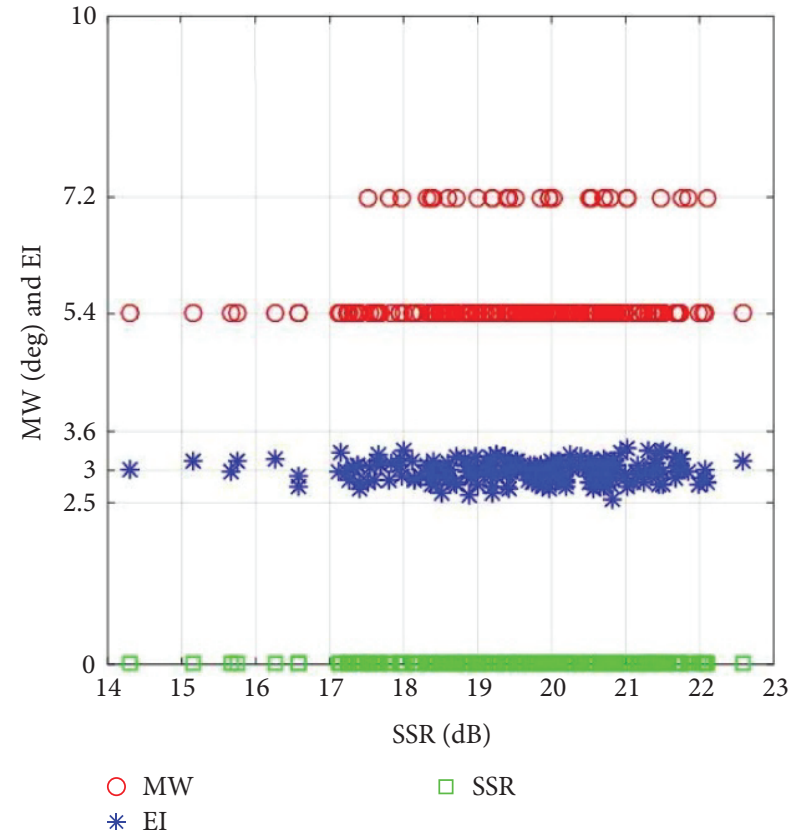

(b)

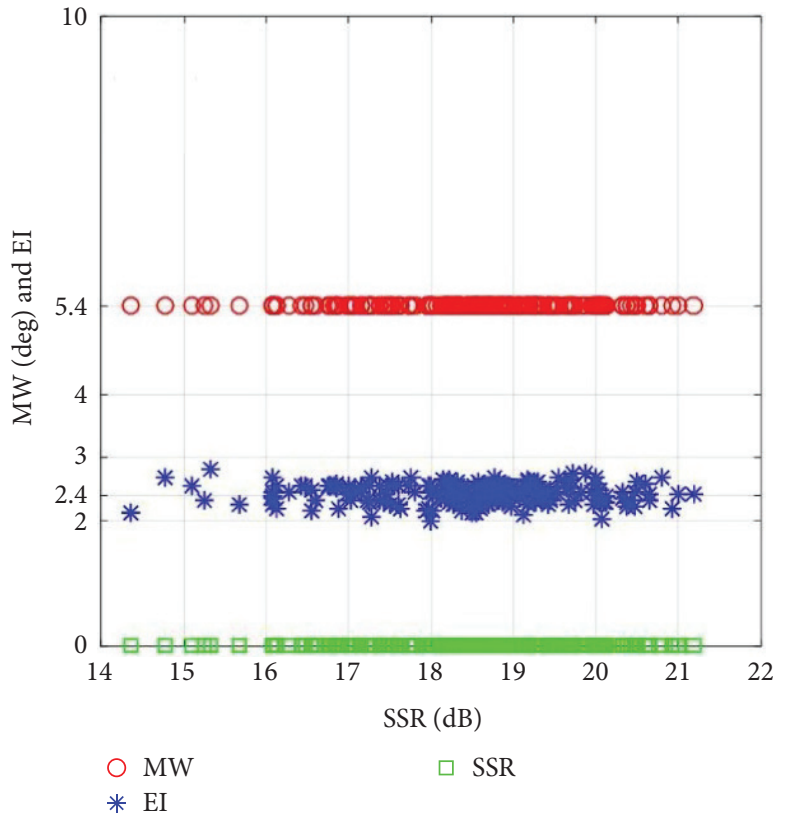

(d)

Figure 6: Evaluation of the partition array performance of four kinds of the partition configurations: (a) $C=7$; (b) $C=9$; (c) $C=8$; (d) $C=16$.

4.3. Source Resolution. The partition array is carried out to assess its usefulness in terms of the resolution. The power of resolution is investigated on two aspects by Matlab random simulation. One is the partition array sources localization, and the other is angle resolution testing. They are checked at four frequencies $(800 \mathrm{~Hz}, 1200 \mathrm{~Hz}, 2000 \mathrm{~Hz}$, and $3000 \mathrm{~Hz}$ ) for two static harmonic sources with the same level at different positions. The positions of two sources are characterized by the azimuth angle and elevation angles: one position is $\left(45^{\circ}, 45^{\circ}\right)$ and the other one is $\left(-45^{\circ},-45^{\circ}\right)$.
In this study, the partition array is optimized, which with the geometry is shown in Figure $7(\mathrm{c})$. The same tests are performed using wheel array and pizza array, which are shown in Figures 7(a) and 7(b), respectively, to compare a source resolution. In each case, white noise is added in the simulated acoustic signals with a signal-to-noise ratio (SNR) of $10 \mathrm{~dB}$ and performs 2000 simulations.

Localized acoustic source maps of partition array are plotted in Figure 9. In the figure, two source positions are depicted using red points on four source maps. Blue portions 


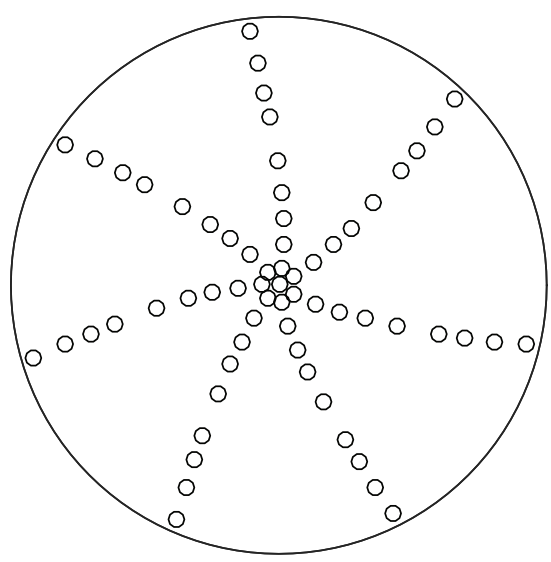

(a)

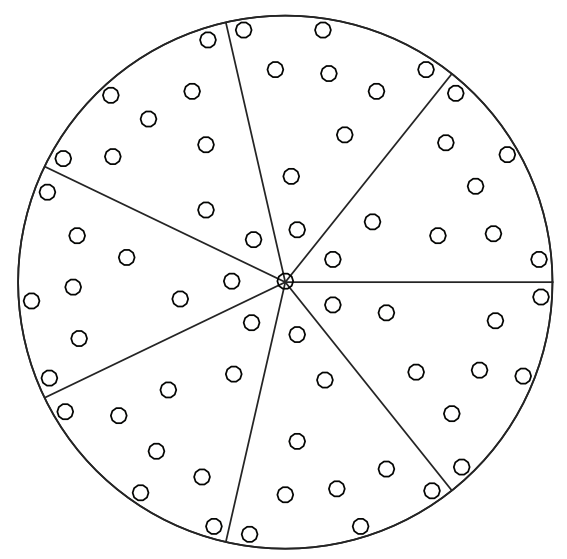

(b)

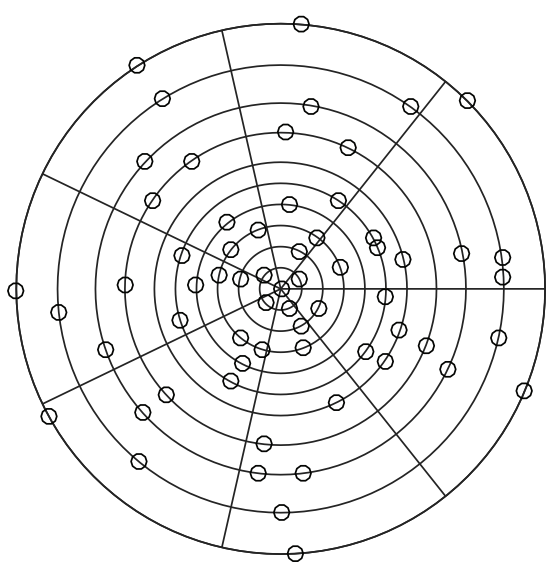

(c)

FiguRE 7: Three different irregular array configurations with the same microphone density and number: (a) log-spiral wheel array; (b) pizza array; (c) partition array.

TABLE 2: Optimal parameters of partition array.

\begin{tabular}{lc}
\hline Parameters & Values \\
\hline Number of subsections & 7 \\
Subsections' microphones & 9 \\
Subsections' grid points & 45 \\
Mainlobe width & 7.2 degree \\
$\begin{array}{l}\text { Maximum sidelobe level } \\
\text { (MSL) }\end{array}$ & $-12 \mathrm{~dB}$ \\
$\begin{array}{l}\text { Sidelobe suppression ratio } \\
\text { (SSR) }\end{array}$ & 21.3 \\
Rings radius & $0.5,1,1.5,2,2.5,3,3.68,4.42,5.3$, \\
Rings' microphones & 6.1 \\
\hline
\end{tabular}

under those red points are the calculated source position based on the measured data of the partition array. The area of the blue portion is approximately proportional to the magnitude of the source. As shown in Figure 9(a), the area of the blue portion is bigger than others in Figures 9(b)-9(d). This is because the measurement at this time is at low frequency $(800 \mathrm{~Hz})$. The main lobe width is wide, and the sidelobe rejection ratio is small. However, as the frequency increases, the array performance keeps getting better and better. That is to say, areas of blue portions get smaller. This phenomenon has a good agreement with the simulation results shown in Figures 6 and 8 . From Figures 9(a)-9(d), we can see that partition array is clearly good in terms of source location accuracy and suppresses the "ghost" of universal source locations. Therefore, it can be concluded that the partition array exhibits a superior ability to separate sources especially for broadband sources.

On the other hand, angle resolution is proposed to further assess the source resolution. The angle resolution is defined by the minimum angle to distinguish the two sources. This procedure is conducted by Matlab simulation. A source is randomly fixed, and the additional source approaches the first source with an angular step size. When the angular step size is less than determined angle, the two sources roll up into one. In this case, the determined angle is the smallest angle. To simplify, we introduce the separation rate to estimate the resolution of three optimized arrays at frequencies of $800 \mathrm{~Hz}, 1200 \mathrm{~Hz}, 2000 \mathrm{~Hz}$, and $3000 \mathrm{~Hz}$, respectively. The simulation results are plotted in Figures 10(a)-10(d). The first source is placed on $\left(45^{\circ}, 45^{\circ}\right)$ of the azimuth and elevation plane. The second source is approaching to the first source with the angular step size of $1.8^{\circ}$.

In Figure 10, the values of $y$-coordinate indicate the percentage of effectual separation of two sources. For example, 1 means $100 \%$ separation of two sources. As we can see from Table 3 , it is the smallest angle to separate two sources of three arrays at different frequencies, and the partition array has the smallest separated angle at all frequencies. Compared with wheel array and pizza array, the partition array has a significant advantage at frequency of $800-2000 \mathrm{~Hz}$ to separate two sources that are close to each other. Furthermore, from Figures 10(a)$10(\mathrm{~d})$, the separation rates of all arrays increase as frequencies increase, especially at $3000 \mathrm{~Hz}$. While all arrays separation rates are higher than ever before, partition array and pizza array have the same smallest separated angle $\left(3.6^{\circ}\right)$. However, the angle separation rate of partition array is higher than that of pizza array. Although the incident wavelength becomes short as the frequency increases, the array aperture becomes larger and the WM becomes narrower. Then, its resolution is improved. The results show that the partition array provides the best source resolution overall the whole bandwidth than the others.

4.4. Doppler Effect Correction. In this part, the partition array is examined in terms of the ability to correct the Doppler effect. Since the Doppler frequency in the de-Dopplerised spectrum is related to the separation ability and variation of the arraypattern, a Matlab simulation is proposed for estimating the emitting frequency of a moving source at the different frequency. We use the bandwidth of the frequency shift around the center frequencies of $800 \mathrm{~Hz}, 1200 \mathrm{~Hz}$, and $2000 \mathrm{~Hz}$ for wheel array, pizza array, and partition array to compare the performance. The source trajectory is at the $7.5 \mathrm{~m}$ distance from the array plane. The position of the source is randomly placed. The source moves with the speed of $120 \mathrm{~km} \mathrm{~h}^{-1}$ along a straight line which is parallel to the source plane. The center of array is placed at point $(16.5,7.5,0)$. Starting point of source movement is the 


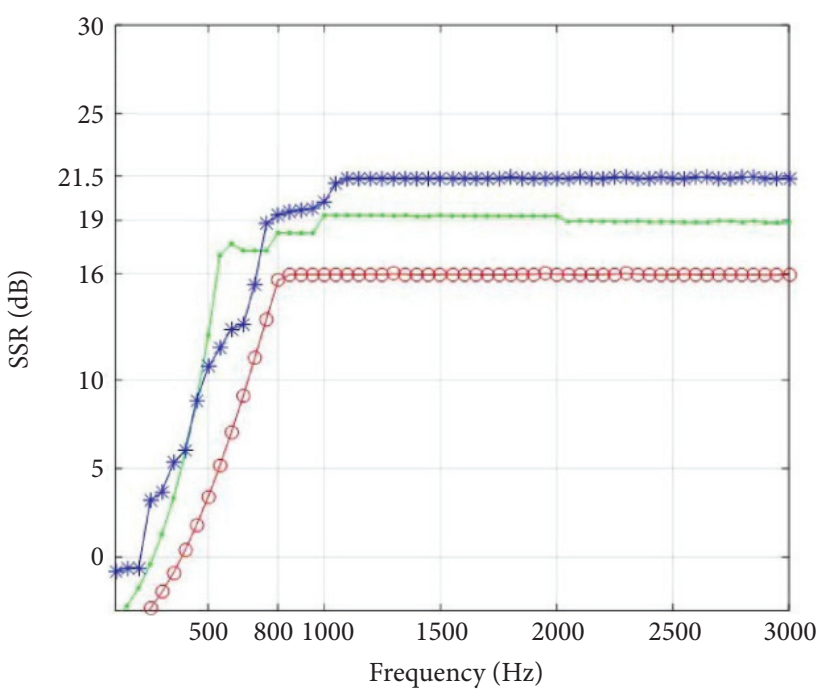

- Wheel

* Partition

(a)

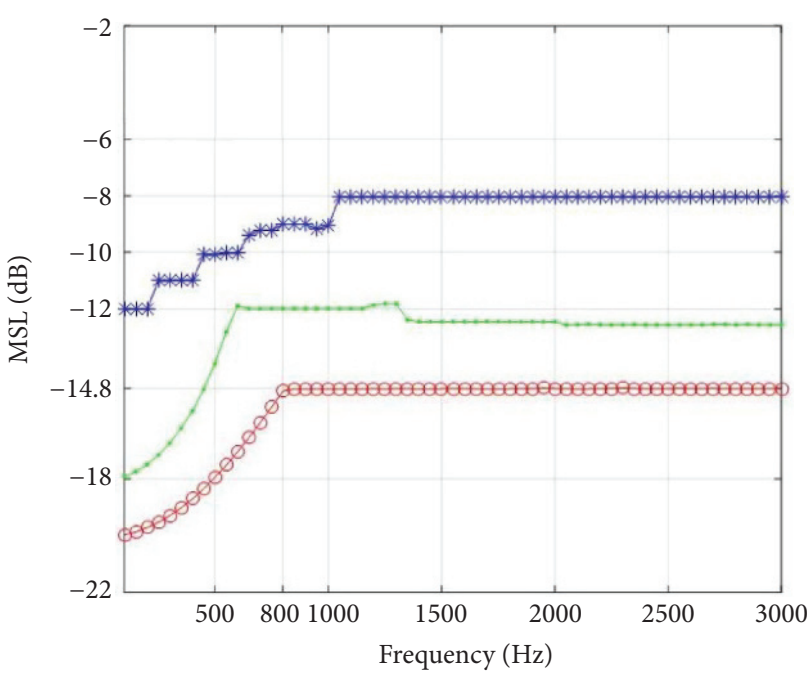

- Wheel

* Partition

(b)

FIGURE 8: Sidelobe level of three different optimized arrays: (a) sidelobe suppression ratio (SSR); (b) maximum sidelobe level (MSL).

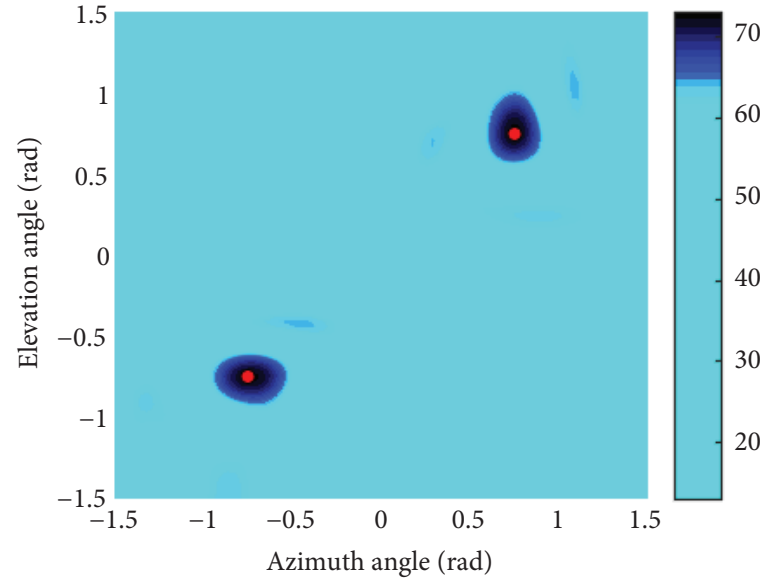

(a)

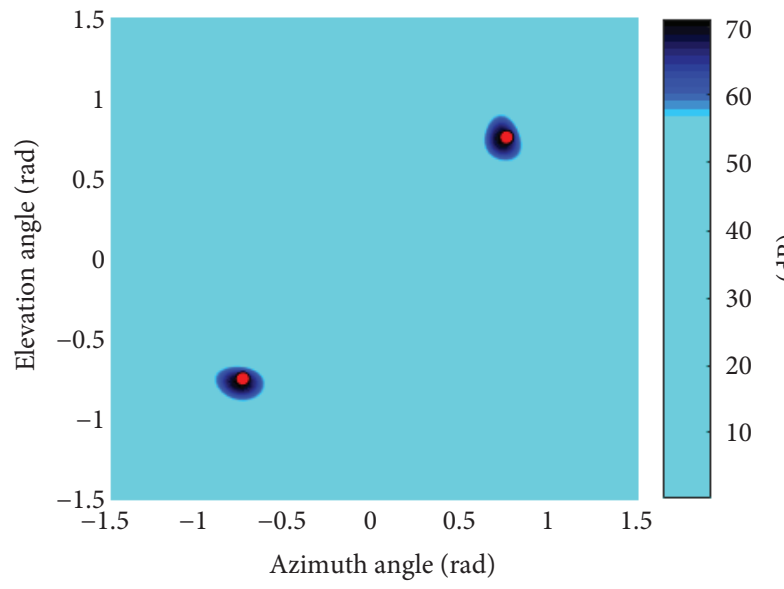

(c)

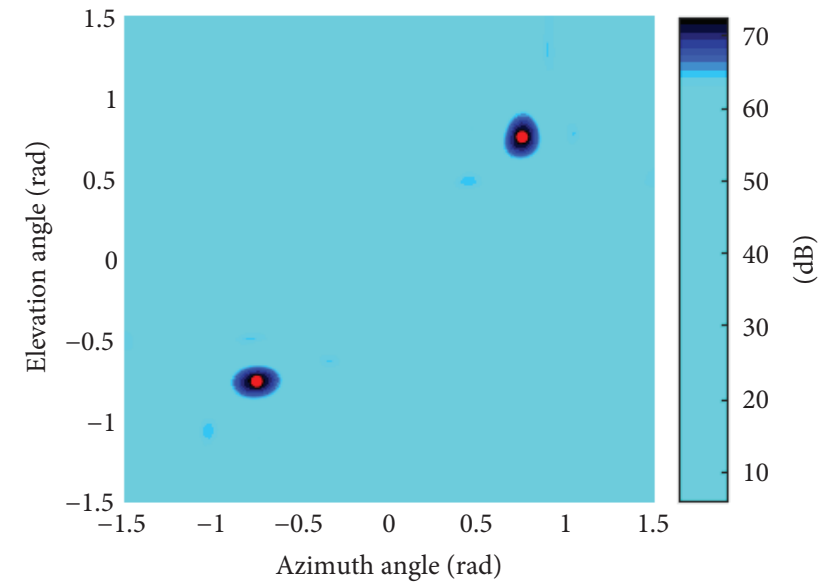

(b)

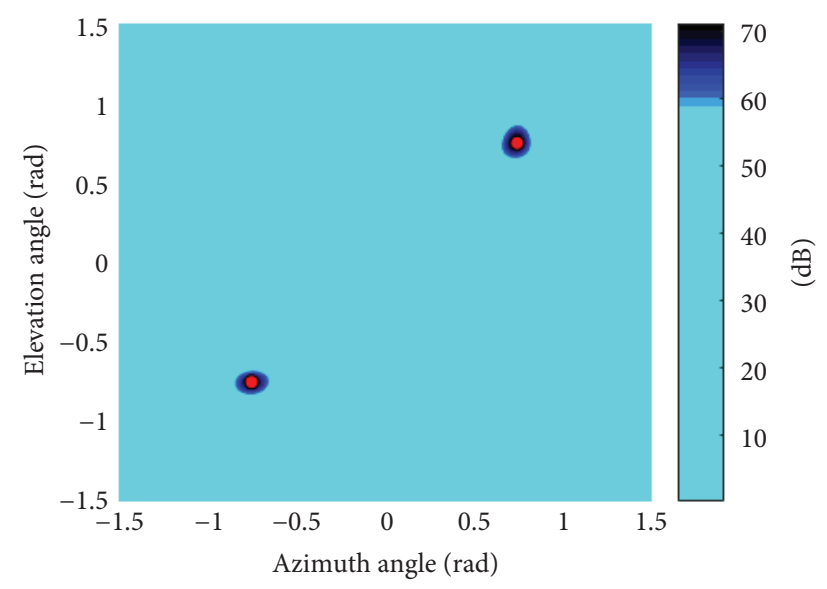

(d)

Figure 9: Acoustic source maps of partition array with different frequencies: (a) $800 \mathrm{~Hz}$; (b) $1200 \mathrm{~Hz}$; (c) $2000 \mathrm{~Hz}$; (d) $3000 \mathrm{~Hz}$. 


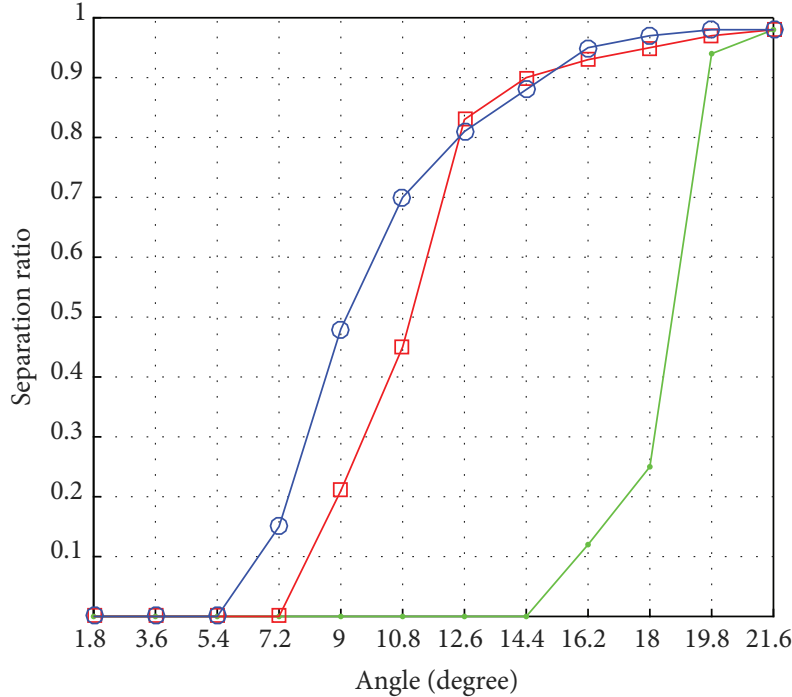

$\rightarrow$ Wheel array $\square$ Pizza array

(a)

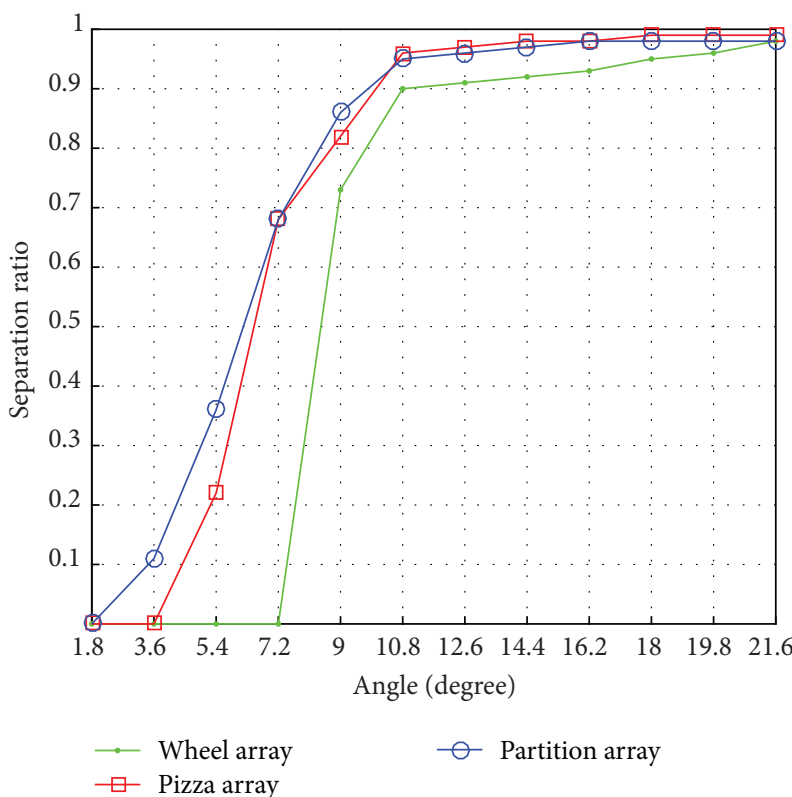

(c)

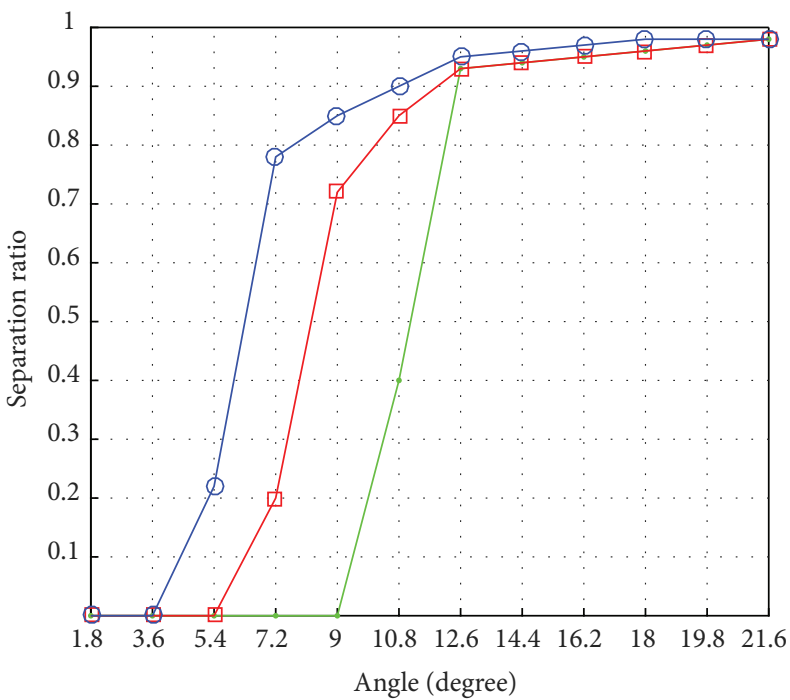

$\longrightarrow$ Wheel array $\square$ Pizza array

(b)

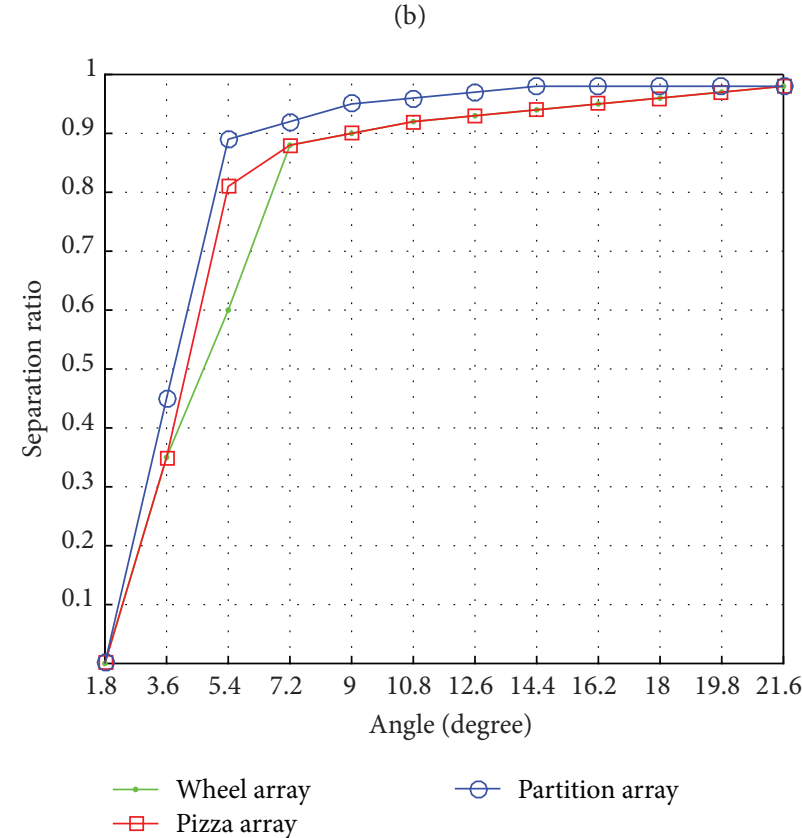

- Wheel array

$\square$ Pizza array

$\oslash$ Partition array

(d)

Figure 10: Angle separation ratio of two sources of the same level with a $10 \mathrm{~dB}$ SNR using three arrays at different frequencies: (a) $800 \mathrm{~Hz}$; (b) $1200 \mathrm{~Hz}$; (c) $2000 \mathrm{~Hz}$; (d) $3000 \mathrm{~Hz}$.

TABLE 3: The smallest separated angle.

\begin{tabular}{lccc}
\hline Frequency $(\mathrm{Hz})$ & Partition array $\left(^{\circ}\right)$ & Pizza array $\left(^{\circ}\right)$ & Wheel array $\left(^{\circ}\right)$ \\
\hline 800 & 7.2 & 9.0 & 18 \\
1200 & 5.4 & 7.2 & 12.6 \\
2000 & 3.6 & 5.4 & 9 \\
3000 & 3.6 & 3.6 & 7.2 \\
\hline
\end{tabular}

coordinate zero point $(0,0,0)$. The signal sample length of microphones is $1 \mathrm{~s}$, and the sampling frequency is $8192 \mathrm{~Hz}$. During signal processing, white noise is added to the simulated acoustic signals with a signal-to-noise ratio (SNR) of $10 \mathrm{~dB}$. In this paper, we use the median spectrum to estimate the frequency shift for wheel array, pizza array, and partition array at frequencies of $800 \mathrm{~Hz}, 1200 \mathrm{~Hz}$, and $2000 \mathrm{~Hz}$, respectively. The results are plotted in Figure 11. 


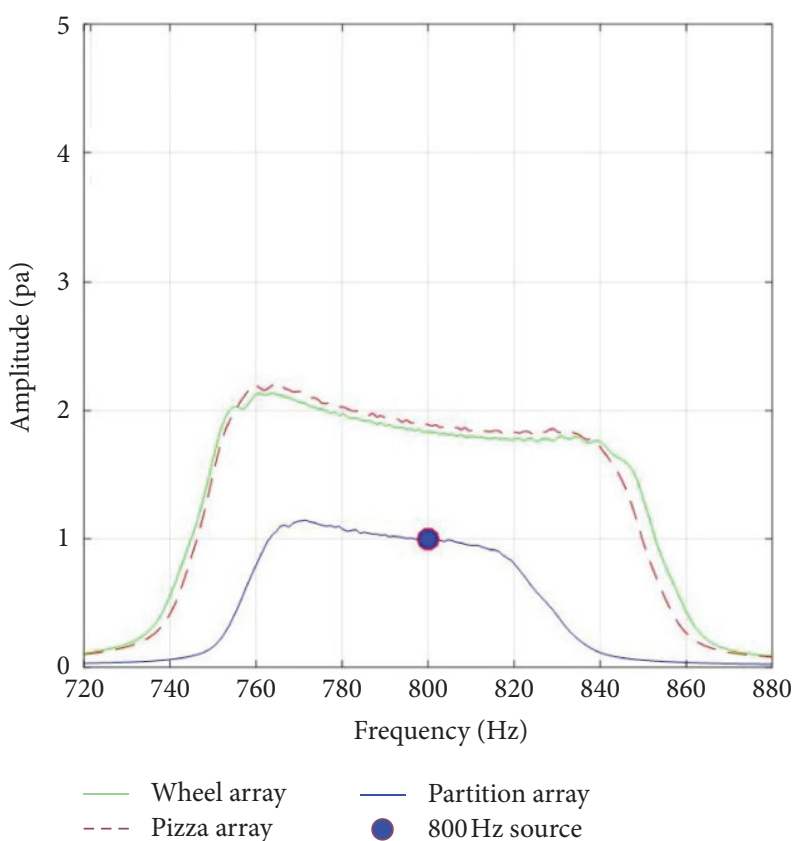

(a)

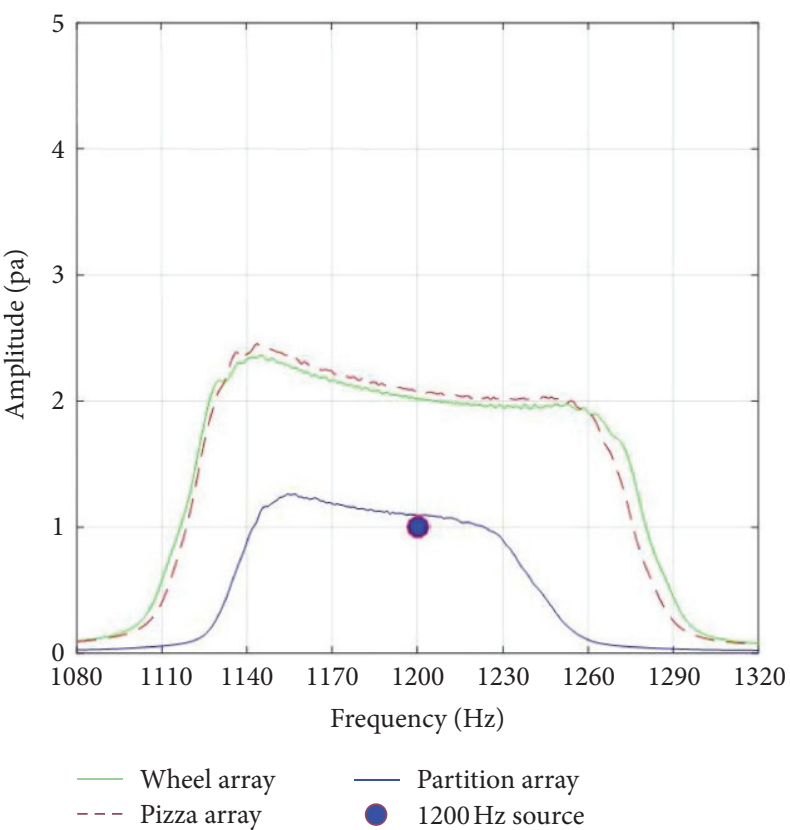

(b)

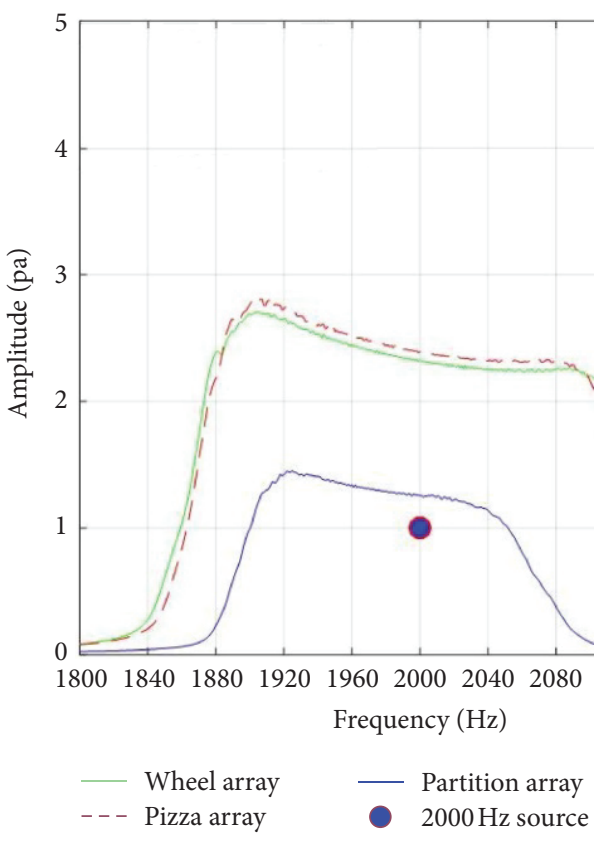

(c)

Figure 11: Median spectrum of three arrays of frequencies: (a) $800 \mathrm{~Hz}$; (b) $1200 \mathrm{~Hz}$; (c) $2000 \mathrm{~Hz}$.

Doppler effect in motion source recognition is reflected in two aspects, which are amplitude change and frequency shift. In order to further evaluate the advantages of the partition array in frequency shift, amplitude correction was made to the collected data in the process of de-Dopplerised, and frequency shift information was retained. The processed results are presented in Figure 11. As can be seen from Figures 11(a)-11(c), the processing results of the partition array have the best consistency with that of the sound source at a different frequency. Although the measurements from other arrays were also processed similarly, their measured amplitude differed greatly from the actual amplitude of the sound source. In addition, to further illustrate this point, the partition array was used to localize one moving source at $800 \mathrm{~Hz}$ with the speed of $120 \mathrm{~km} \cdot \mathrm{h}^{-1}$. The acoustic maps at different seconds are shown in Figure 12. In Figures 12(a)-12(d), the red points depict the source positions at every second, and blue portions under each red point are the calculated source position 


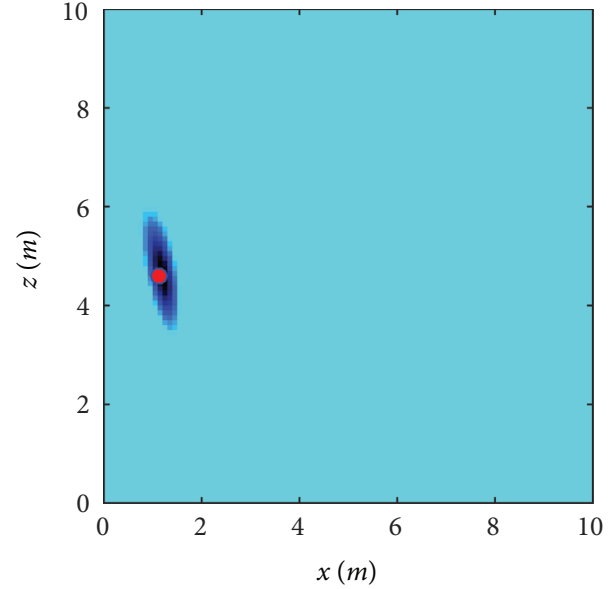

(a)

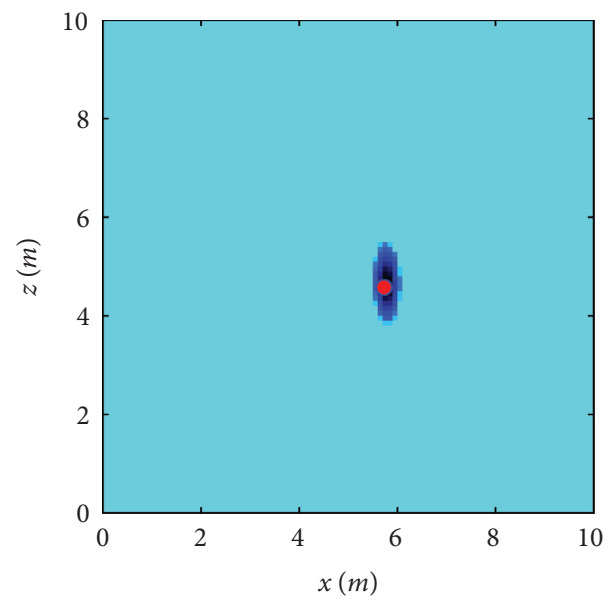

(c)
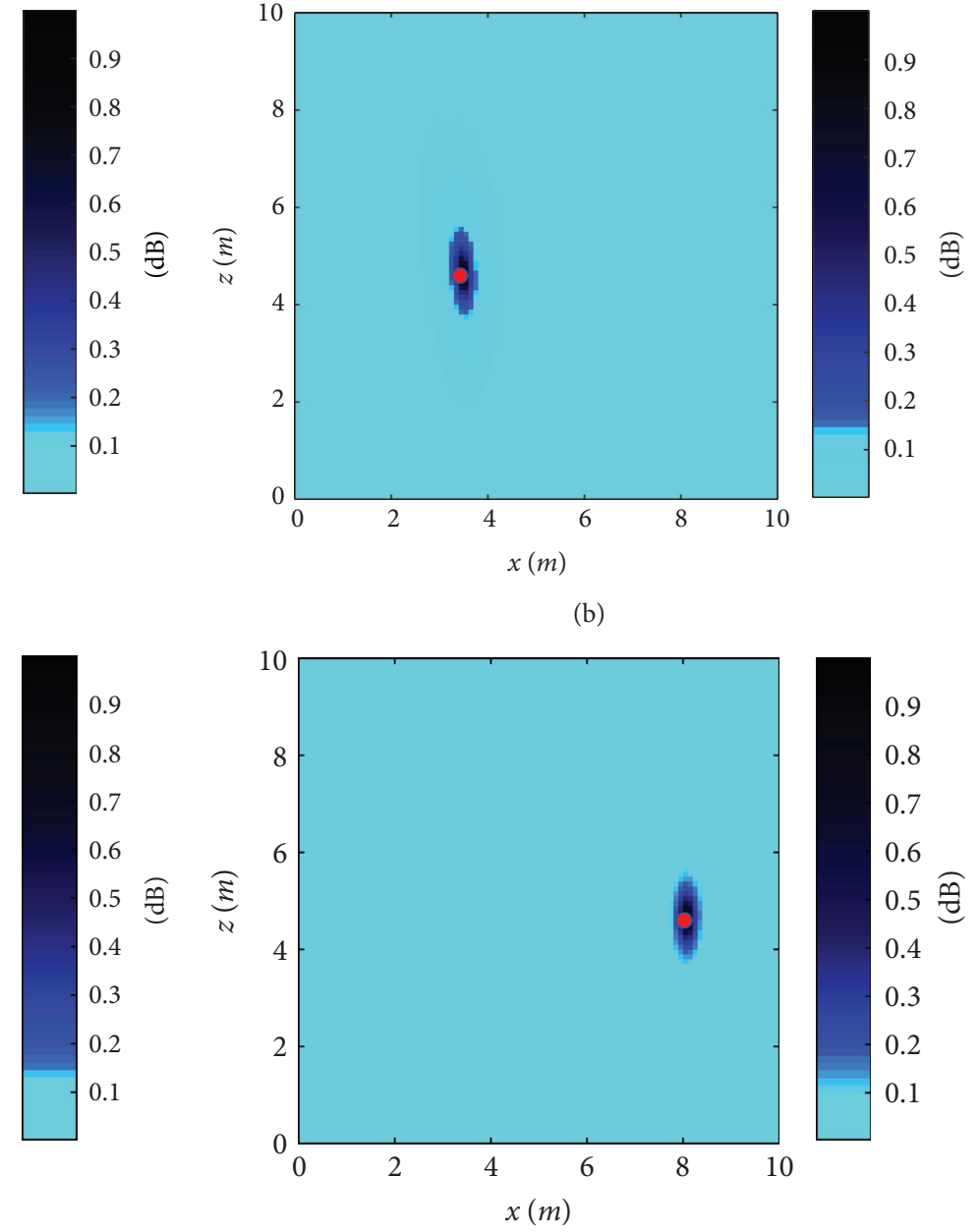

(d)

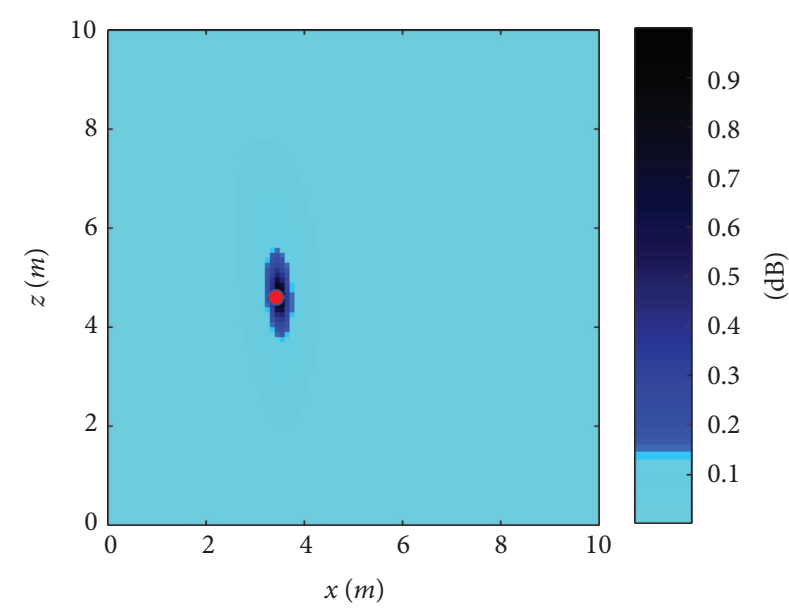

(b)

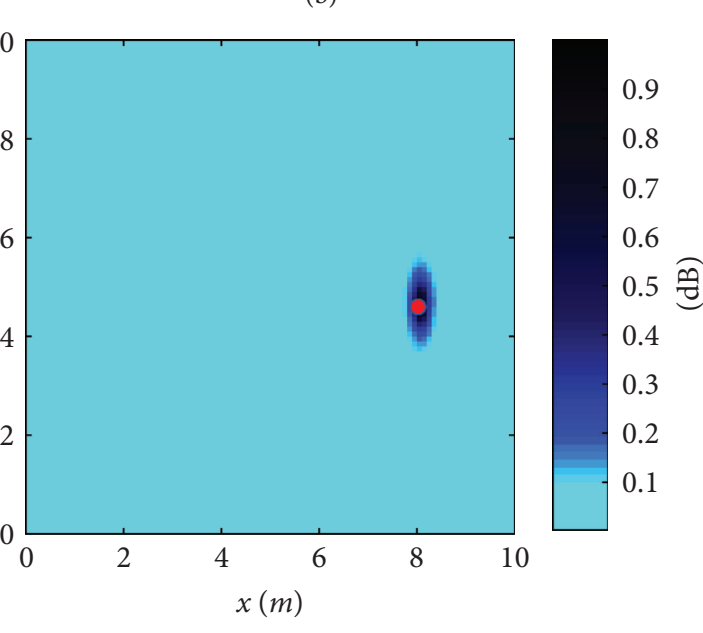

FIGURe 12: Acoustic maps of partition array in different seconds: (a) $0.47 \mathrm{~s}$; (b) $0.49 \mathrm{~s}$; (c) $0.51 \mathrm{~s}$; (d) $0.53 \mathrm{~s}$.

based on the processing data of the partition array. The area of the blue portion is approximately proportional to the magnitude of the source. At every second, the partition array exhibits a good source position fit. This reflects the invariance of the array beampattern. From this point of view, the partition array has the merit of measuring the moving sound source.

On the other hand, the median spectrum is in place to estimate array performance on the aspect of the frequency shift. Here, the median spectrum is characterized as the average value of the processing spectrums. As shown in Figures 11(a)-11(c), the partition array has the narrowest frequency shift bandwidth at every frequency. When compared with the wheel array and pizza array, the frequency shift width of the partition array decreases by about $40 \%$ of their shift width. The wheel array and pizza array have almost the same characteristics in the frequency shift. The results show that the partition array configuration optimization may enhance the invariance of array beampattern and a better correction of the Doppler effect in the measure of motion source.

\section{Experiments}

In this section, we have conducted experiments to validate the proposed array configuration method. The experimental setup photo is shown in Figure 13(a). A partition array was set up $7.5 \mathrm{~m}$ from the car surface. The array center is at point $(16.5,0,1.2)$. The $x$-axis is horizontal, parallel to the line along with the car moved, the $y$-axis is vertical, pointing downward to the ground, and the $z$-axis is horizontal, pointing toward the car.

Figure 13(b) shows the microphone array in detail. Microphone array configuration was built based on the simulation results in Section 4. There are 64 microphones and 10 rings, and each ring radius was the same as the optimal partition array shown in Figure $7(\mathrm{c})$. The array aperture is $2 \mathrm{~m}$. A microphone was placed at the center of the array.

During the measurement, the wind speed was less than $3 \mathrm{~m} \mathrm{~s}^{-1}$, and there was no rain or snow. The sample frequency was $8192 \mathrm{~Hz}$, and the sampling length was $1 \mathrm{~s}$. The data were processed with a moving focus beamforming 


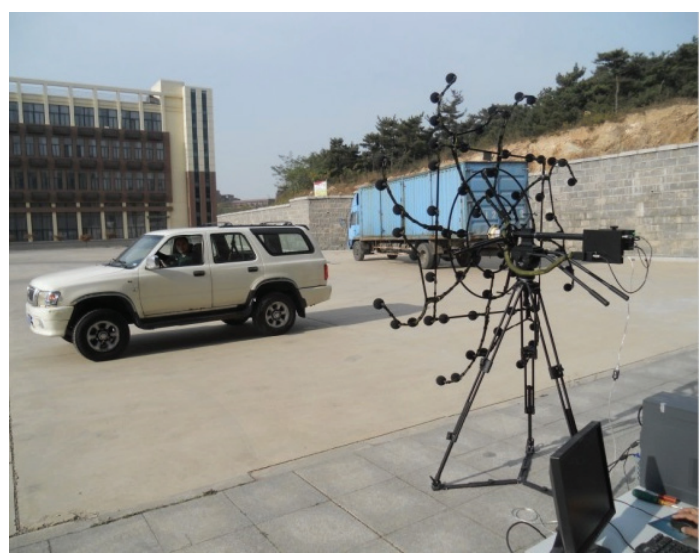

(a)

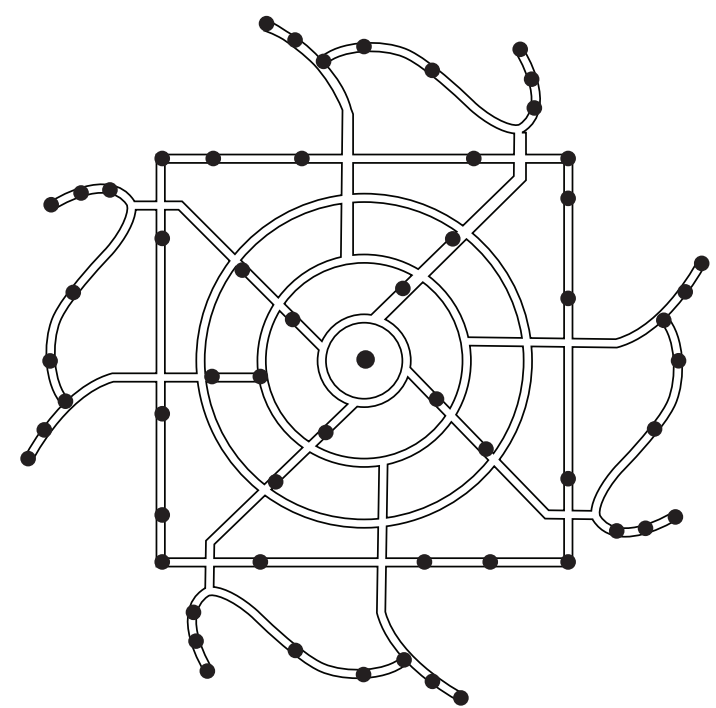

(b)

FiguRE 13: Experimental setup: (a) experimental photo; (b) microphone array.

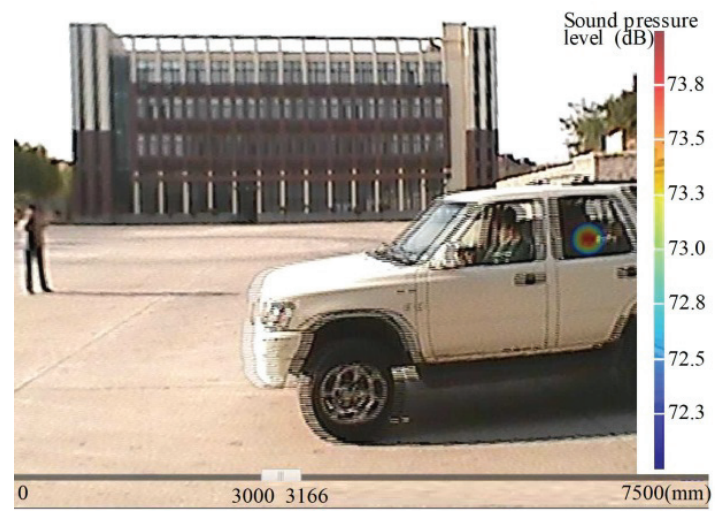

(a)

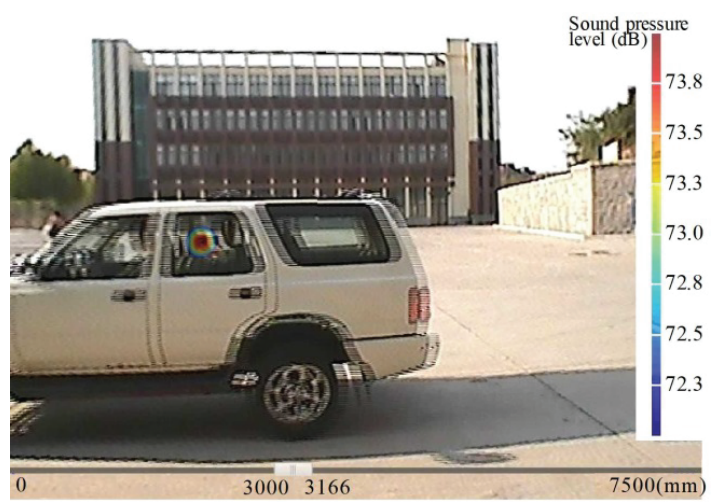

(c)

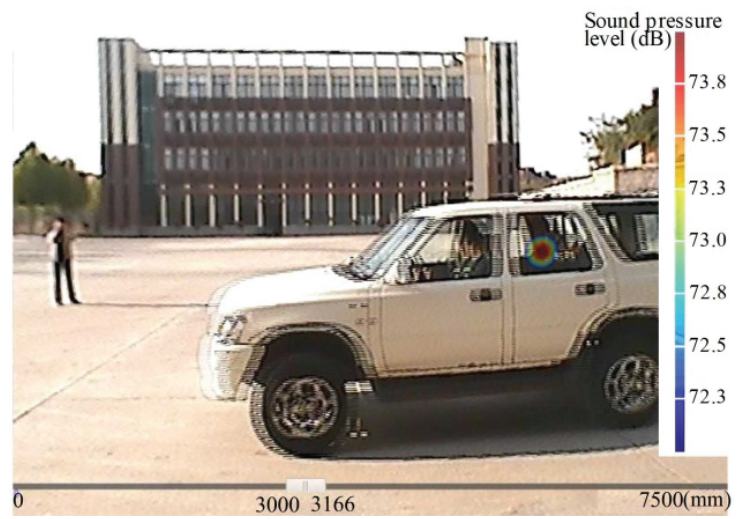

(b)

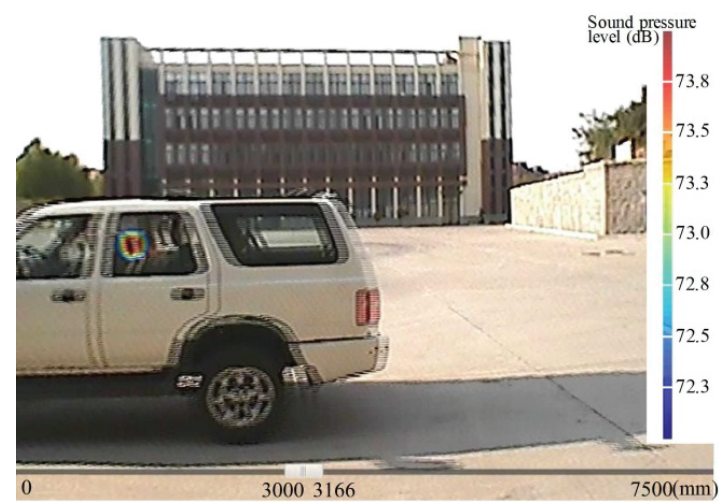

(d)

FIgURe 14: Acoustic maps of partition array with different time: (a) $0.47 \mathrm{~s}$; (b) $0.49 \mathrm{~s}$; (c) $0.51 \mathrm{~s}$; (d) $0.53 \mathrm{~s}$.

algorithm. This algorithm was based on the assumption that a source was moving in front of the array of microphones on an arbitrary trajectory with variable speed. The basic idea was to focus on an assumed source position on reconstruction plane.
There are two important experiments. Firstly, the examination of the localization of one moving source at $800 \mathrm{~Hz}$ with the speed of $120 \mathrm{~km} \cdot \mathrm{h}^{-1}$ using partition array. A loudspeaker was placed in a moving car near the left rear side window, which is generating $800 \mathrm{~Hz}$ tone and the amplitude 


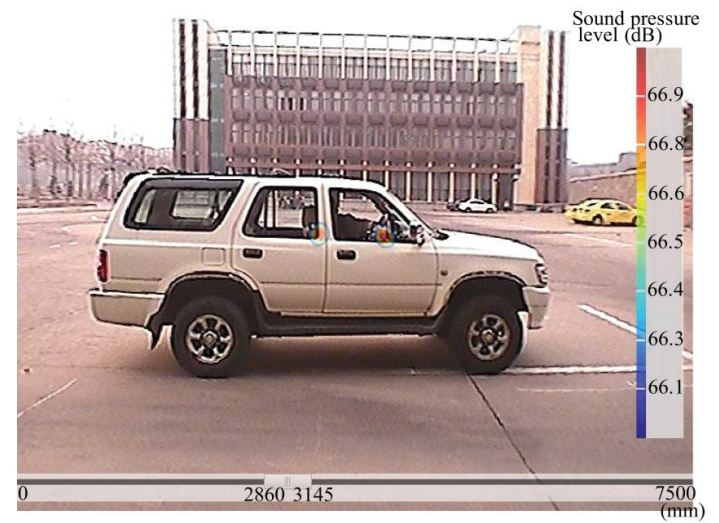

FIgURe 15: Acoustic map of two closer sources.

is $74 \mathrm{~dB}$. Figure 14 shows localization results for loudspeaker at different moment.

In Figure 14, the color maps are utilized to show the amplitude variation from $72 \mathrm{~dB}$ to $74 \mathrm{~dB}$, which corresponds to color scale from blue to red. As can be observed in the parts (a) to (d) of Figure 14, the areas of acoustic maps are small. It covers the loudspeaker almost completely at every second. Moreover, the maps are quite clear and not "ghost" sources appeared. This means that acoustic maps can focus on the source position accurately. Compared with the numerical simulating results in Figure 12, the results of moving source localization have a good agreement at the $800 \mathrm{~Hz}$.

Secondly, to assess the resolution of the partition array, two loudspeakers were placed in a moving car near the left front and rear side window, respectively. The distance between them is the smallest separated angle. It is $3.6^{\circ}$ at high frequencies of $3000 \mathrm{~Hz}$, which is shown in Table 3. Within a meter scale, $3.6^{\circ}$ equals to $0.0628 \mathrm{~m}$. Loudspeakers generate two sound waves with the same frequency of $3000 \mathrm{~Hz}$ and sound pressure level of $67 \mathrm{~dB}$. The localization results are shown in Figure 15. As showed in the figure, the acoustic map areas of two sources are separated and not "ghost" sources appeared around them. The maps cover the loudspeakers almost completely. It is demonstrated that the partition array can locate and separate two closer sources clearly.

These experiments have highlighted that the localization of moving sources can be improved using partition array. Interference has been eliminated, and the localization seems more accurate. The experiment results verified the performance found on the simulation.

\section{Conclusions}

A novel irregular array configuration method by utilizing partition filtering was put forward in this paper. Partition filtering conditions, which takes into account the sound field characteristics and array properties, were determined to quantify the relationship between the array configuration and performance. Those relations are significant to resolve the inevitable variance of array beampattern and the Doppler effect, especially for making moving wideband sources measure possible. The problem of array configuration was formulated into position matrix determinants and solved fast by employing the adaptive partition algorithm.

This method involves many practical advantages. Adaptive partition procedure and quantitative relations help to preselect array configurations. This can significantly reduce the computation speed, and it is more suitable for array build especially large number microphones. Array irregular layout can effectively extend array aperture and reduce redundant spatial samplings to achieve superior array performance, such as high resolution (narrowest MW) and interference suppression (the larger MSL and SSR) by adjusting grid spacings and partition number. Meanwhile, the array configuration can be easily generated with optimal MW, MSL, and SSR characteristics for a specific acoustic test. It is fitted to achieve high quality beamformer outputs for a small-scale array over a wideband. In addition, since partition filtering can sufficiently capture the spatial variation of beampattern by an adaptive procedure, the partition array has stronger capacity of Doppler effect correction. It is more suitable for the localization of moving sources.

\section{Data Availability}

The data used to support the findings of this study are available from the corresponding author upon request.

\section{Conflicts of Interest}

The authors declare that they have no conflicts of interest regarding the publication of this paper.

\section{Acknowledgments}

This research was funded by the National Natural Science Foundation of China, grant nos. 61871447, 61671262, and 51806114 .

\section{References}

[1] H. Kook, G. B. Moebs, P. Davies, and J. S. Bolton, "An efficient procedure for visualizing the sound field radiated by vehicles during standardized passby tests," Journal of Sound and Vibration, vol. 233, no. 1, pp. 137-156, 2000.

[2] J. Zhang, G. Squicciarini, and D. J. Thompson, "Implications of the directivity of railway noise sources for their quantification using conventional performing," Journal of Sound and Vibration, vol. 459, pp. 1-23, 2019.

[3] M. Genescà, J. Romeu, T. Pàmies, and A. Sánchez, "Real time aircraft fly-over noise discrimination," Journal of Sound and Vibration, vol. 323, no. 2, pp. 112-129, 2009.

[4] P. Castellini, A. Sassaroli, A. Paonessa, A. Peiffer, and A. Roeder, "Average beamforming in reverberant fields: application on helicopter and airplane cockpits," Applied Acoustics, vol. 74, no. 1, pp. 198-210, 2013.

[5] C. C. Pagani, D. S. Souza, and M. A. F. Medeiros, "Experimental investigation on the effect of slat geometrical configurations on aerodynamic noise," Journal of Sound and Vibration, vol. 394, pp. 256-279, 2017. 
[6] G. Herold and E. Sarradj, "Microphone array method for the characterization of rotating sound sources in axial fans," Noise Control Engineering Journal, vol. 63, no. 6, pp. 546-551, 2015.

[7] C. Q. Zhang, Z. Y. Gao, Y. Y. Chen et al., "Locating and tracking sound sources on a horizontal axis wind turbine using a compact microphone array based on beamforming," Applied Acoustics, vol. 146, pp. 295-309, 2019.

[8] W. Ma, H. Bao, C. Zhang et al., "Beamforming of phased microphone array for rotating sound source localization," Journal of Sound and Vibration, vol. 467, Article ID 115064, 2020.

[9] P. Chiariotti, M. Martarelli, and P. Castellini, "Acoustic beamforming for noise source localization - reviews, methodology and applications," Mechanical Systems and Signal Processing, vol. 120, no. 1, pp. 422-448, 2019.

[10] B. P. Kumar and G. R. Branner, "Design of unequally spaced arrays for performance improvement," IEEE Transactions on Antennas and Propagation, vol. 47, no. 3, pp. 511-523, 1999.

[11] B. P. Kumar and G. R. Branner, "Generalized analytical technique for the synthesis of unequally spaced arrays with linear, planar, cylindrical or spherical geometry," IEEE Transactions on Antennas and Propagation, vol. 53, no. 2, pp. 621-634, 2005.

[12] D. Yang, Z. Wang, B. Li, Y. Luo, and X. Lian, "Quantitative measurement of pass-by noise radiated by vehicles running at high speeds," Journal of Sound and Vibration, vol. 330, no. 7, pp. 1352-1364, 2011.

[13] F. Martellotta, "On the use of microphone arrays to visualize spatial sound field information," Applied Acoustics, vol. 74, no. 8, pp. 987-1000, 2013.

[14] J. A. Ballesteros, E. Sarradj, M. D. Geyer, and M. J. Ballesteros, "Noise source identification with beamforming in the pass-by of a car," Applied Acoustics, vol. 93, pp. 106-119, 2015.

[15] J. Yu and K. D. Donohue, "Optimal irregular microphone distributions with enhanced beamforming performance in immersive environments," The Journal of the Acoustical Society of America, vol. 134, no. 3, pp. 2066-2077, 2013.

[16] X. Wang, M. Amin, and X. Wang, "Robust sparse array design for adaptive beamforming against DOA mismatch," Signal Processing, vol. 146, pp. 41-49, 2018.

[17] D. H. Johnson and D. E. Dudgeon, Array Signal Processing Concepts and Techniques, Prentice-Hall, Upper Saddle River, Bergen, 1st edition, 1993.

[18] H. Kook, P. Davies, and J. S. Bolton, "Statistical properties of random sparse arrays," Journal of Sound and Vibration, vol. 255, no. 5, pp. 819-848, 2002.

[19] J. J. Christensen and J. Hald, "Acoustics and vibrationsmeasurements-beamforming," Bruel \& Kjaer Technical Review, vol. 1, no. 1, 2004.

[20] S. Gade and J. Hald, Array Designs Optimized for Both LowFrequency NVH and High-Frequency Beamforming, SAE Technical Paper-SAE International, Warrendale, PA, USA, 2005.

[21] L. D. Val, M. Jiménez, A. Izquierdo, and J. Villacorta, “Optimisation of sensor positions in random linear arrays based on statistical relations between geometry and performance," Applied Acoustics, vol. 73, pp. 78-82, 2012.

[22] R. P. Dougherty, "Spiral-shaped array for broadband imaging," U.S. Patent No. 5, 1998.

[23] D. Zhao, X. Liu, W. Chen, and Y. Chen, "Optimized design for sparse cross arrays in both near-field and far-field," IEEE Journal of Oceanic Engineering, vol. 44, no. 3, pp. 783-795, 2019.
[24] E. Arcondoulis and Y. Liu, "An iterative microphone removal method for acoustic beamforming array design," Journal of Sound and Vibration, vol. 442, pp. 552-571, 2019.

[25] M. Aldeman and G. Raman, "Effects of array scaling and advanced beamforming algorithms on the angular resolution of microphone array systems," Applied Acoustics, vol. 132, pp. 58-81, 2018.

[26] L. C. Florent, J. H. Thomas, F. Poisson, and J. C. Pascal, "Genetic optimisation of a plane array geometry for beamforming-application to source localisation in a high speed train," Journal of Sound and Vibration, vol. 233, no. 1, pp. 137-156, 2015.

[27] V. T. Rathod, "A review of acoustic impedance matching techniques for piezoelectric sensors and transducers," Sensors, vol. 20, no. 14, p. 4051, 2020.

[28] X. Zhang, Y. Zhang, and C. Lu, "Flow and noise characteristics of centrifugal fan in low pressure environment," Processes, vol. 8 , no. 8 , p. $985,2020$. 